\title{
Coastal Community Resiliency Enhancement Using Marine Hydrokinetic (MHK) Resources and Networked Microgrids
}

Architecture, Optimization, and Control

Energy Systems Division 


\begin{abstract}
About Argonne National Laboratory
Argonne is a U.S. Department of Energy laboratory managed by UChicago Argonne, LLC under contract DE-AC02-06CH11357. The Laboratory's main facility is outside Chicago, at 9700 South Cass Avenue, Lemont, Illinois 60439. For information about Argonne and its pioneering science and technology programs, see www.anl.gov.
\end{abstract}

\title{
DOCUMENT AVAILABILITY
}

Online Access: U.S. Department of Energy (DOE) reports produced after 1991 and a growing number of pre-1991 documents are available free at OSTI.GOV (http://www.osti.gov/),

a service of the US Dept. of Energy's Office of Scientific and Technical Information.

Reports not in digital format may be purchased by the public from the National Technical Information Service (NTIS):

U.S. Department of Commerce

National Technical Information Service

5301 Shawnee Road

Alexandria, VA 22312

www.ntis.gov

Phone: (800) 553-NTIS (6847) or (703) 605-6000

Fax: (703) 605-6900

Email: orders@ntis.gov

Reports not in digital format are available to DOE and DOE contractors from the Office of Scientific and Technical Information (OSTI):

U.S. Department of Energy

Office of Scientific and Technical Information

P.O. Box 62

Oak Ridge, TN 37831-0062

www.osti.gov

Phone: (865) 576-8401

Fax: (865) 576-5728

Email: reports@osti.gov

\section{Disclaimer}

This report was prepared as an account of work sponsored by an agency of the United States Government. Neither the United States Government nor any agency thereof, nor UChicago Argonne, LLC, nor any of their employees or officers, makes any warranty, express or implied, or assumes any legal liability or responsibility for the accuracy, completeness, or usefulness of any information, apparatus, product, or process disclosed, or represents that its use would not infringe privately owned rights. Reference herein to any specific commercial product, process, or service by trade name, trademark, manufacturer, or otherwise, does not necessarily constitute or imply its endorsement, recommendation, or favoring by the United States Government or any agency thereof. The views and opinions of document authors expressed herein do not necessarily state or reflect those of the United States Government or any agency thereof, Argonne National Laboratory, or UChicago Argonne, LLC. 


\section{Coastal Community Resiliency Enhancement Using Marine Hydrokinetic (MHK) Resources and Networked Microgrids}

Architecture, Optimization, and Control

by

Yuhua Du¹, Yuxi Men¹, Xiaonan Lu1, Jianzhe Liu², Feng Qiu², and Bo Chen²

${ }^{1}$ College of Engineering, Temple University

${ }^{2}$ Center for Energy, Environmental, and Economic Systems Analysis, Energy Systems Division, Argonne National Laboratory

December 2020 



\section{CONTENTS}

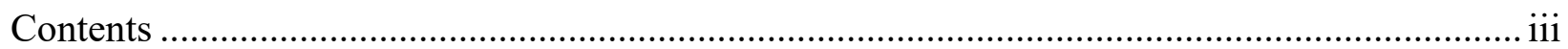

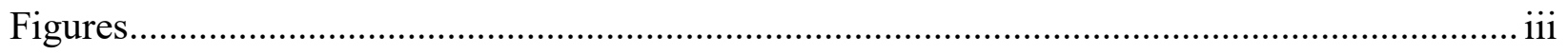

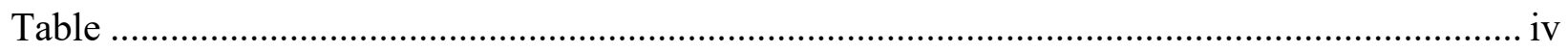

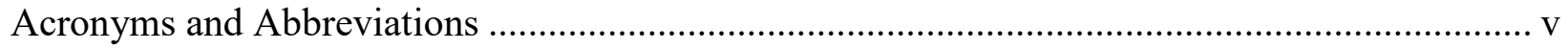

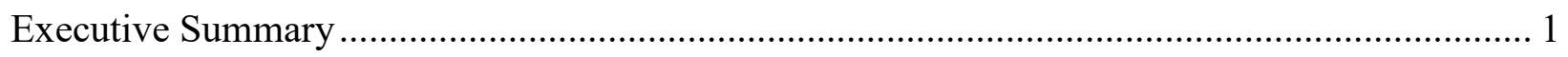

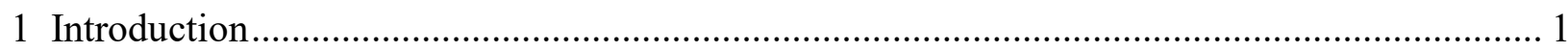

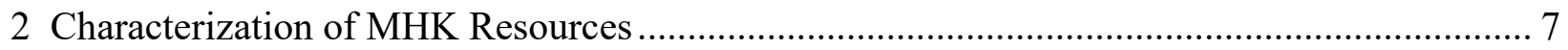

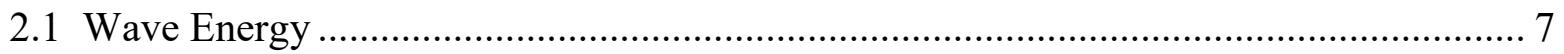

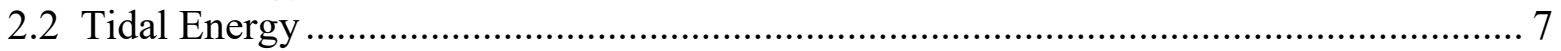

3 Coastal Networked MG Implementation Surrounding MHK Resources .................................. 9

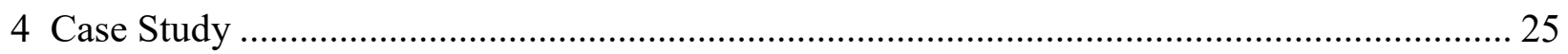

4.1 Case I: Coastal Distribution Feeder Reconfiguration with Positive Tidal Generation......................................................................................... 26

4.2 Case II: Coastal Distribution Feeder Reconfiguration with

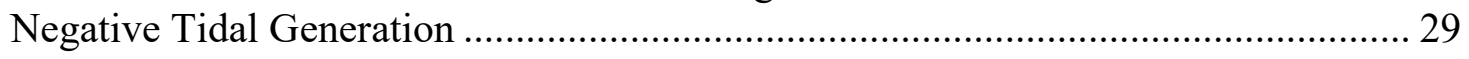

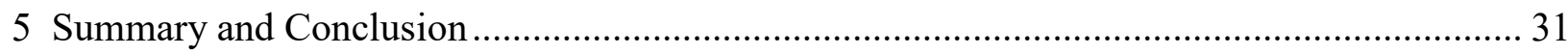

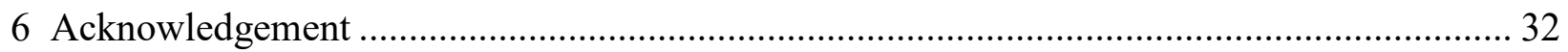

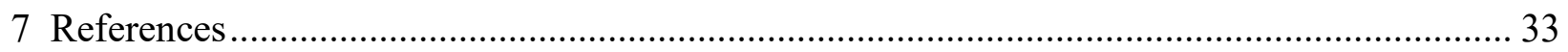

\section{FIGURES}

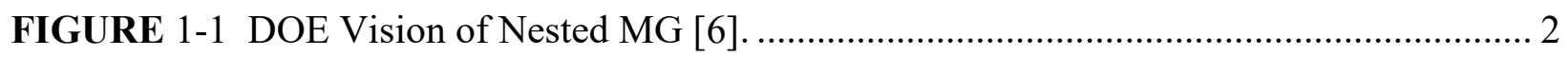

FIGURE 1-2 Comparison between (a) Conventional Networked MGs and (b) Dynamic MGs. . 4

FIGURE 2-1 Sample Wave Generation Profile....................................................................... 7

FIGURE 2-2 Sample Tidal Current Profile with Respect to Moon Phases...................................... 8

FIGURE 3-1 Conventional Structure of an Inverter-based DG [23] ........................................ 12

FIGURE 3-2 Dynamic MG Control Framework for Coastal Feeder. ………………….............. 14

FIGURE 3-3 Two-stage Restoration Strategy for Coastal Feeder Using Dynamic MGs. ......... 24

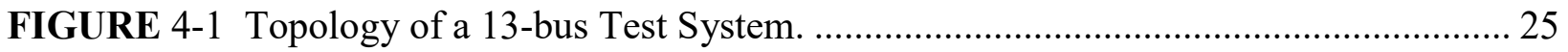




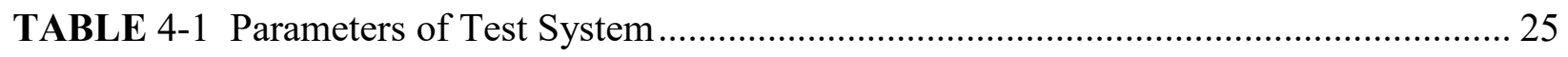

FIGURE 4-2 Dynamic MG Reconfiguration Schemes. .................................................. 26

FIGURE 4-3 Simulation Results for System Reconfiguration in Case I............................... 28

FIGURE 4-4 Simulation Results for System Reconfiguration in Case II. .............................. 30

\section{TABLE}

4-1 Parameters of Test System 


\section{ACRONYMS AND ABBREVIATIONS}

BESS battery energy storage sources

CHP combined heat and power

CPS cyber-physical systems

DG distributed generator

IED intelligent electronic device

MG microgrid

Min-MG minimum MG

MHK marine hydrokinetic

NC normally closed

NO normally open

PCC point of common coupling

POI point of interconnection

PV photovoltaic

SSW smart switch

WEC wave energy converter 


\section{EXECUTIVE SUMMARY}

Enormous challenges are inherent in the operation of modern distribution grids. In particular, distribution feeders can be severely compromised under extreme conditions such as large-scale weather events (e.g., hurricanes, floods, and other types of natural disasters). To combat this problem, they turn to local energy support from distributed energy resources, such as solar and wind, marine hydrolinetic (MHK), and other types of energy.

In this report, we investigate the actual characteristics of MHK resources to enhance coastal community resiliency. Two typical types of MHK resources (i.e., wave and tidal resources) illustrate the coastal resilience enhancement framework. By identifying their operational characteristics, such as target time-scales and potential power generation variations, we detail a holistic coastal community resiliency enhancement framework that integrates both optimization and control efforts.

The optimization problem is formulated to significantly minimize the power outage time at the critical infrastructures in coastal communities by implementing multiple autonomous and dynamic microgrids around MHK resources. Therefore, during severe power outages caused by natural disasters (e.g., floods, hurricanes), the coastal community load profiles can be satisfied using local MHK resources in each microgrid with limited or no power support from upstream power grids.

To ensure operational continuity for coastal communities, we also developed distributed and resilient control schemes at the MHK asset level in coordination with system-level reconfiguration plans to enable the formation of autonomous and dynamic microgrids. We develop a dynamic consensus algorithm to achieve resilient frequency and voltage control within each autonomous microgrid to guarantee that local loads, especially critical loads, are energized with stability guarantees. Note that the designed distributed control schemes only rely on neighboring and sparse communication networks, without any dedicated central communication systems; therefore, operational resiliency can be further enhanced using localized and released communication requirements. Additional coastal community operational functions are also integrated into the designed control schemes, such as rational power distribution that accounts for real-time MHK power generation.

Based on the developed coastal community resiliency enhancement framework, we also developed a conceptual black start strategy, leveraging MHK-enabled autonomous and dynamic microgrids. This black start strategy features a multiphase configuration to restore grid services and load energization along coastal distribution feeders. This conceptual multiphase black start framework is an emerging approach that can drastically enhance coastal community resiliency, even in the extreme conditions of coastal distribution grid blackouts.

The optimization and distributed control algorithms implemented in this technical report suggest a feasible solution of enhancing distribution grid resiliency with local distributed generation. 
This page intentionally blank.

ES-2 


\section{INTRODUCTION}

The growing world population and the continued industrialization of developing countries are producing increasing levels of energy use. Solar and wind energy, and marine and hydrokinetic (MHK) resources, among others, have significant potential for electrical power generation. In particular, wave energy, tidal energy, and ocean currents contain most of the ocean's kinetic energy, and MHK energy technologies can convert some of this kinetic energy to generate electrical power. The power provided by MHK energy has the potential to provide a significant amount of the national energy demand in the United States. MHK energy resources can reduce dependence on conventional energy sources, supply clean energy to coastal communities, and create job opportunities [1], [2].

When an event such as a power outage leaves communities isolated from the main power grid, MHK resources can provide essential support. Based on optimal dispatch and control, the balance between generation and demand can always be ensured by turning circuit breakers on and off. Moreover, wave and tidal energy are commonly accessible among coastal communities; integrating them into their energy portfolio would therefore be helpful in balancing available sources of energy from other types of resources.

It is critical to conduct research about the operational resiliency of coastal communities. A coastal community could have limited access to large substations, and thus be vulnerable to system contingencies; however, coastal communities are rich in offshore resources, which promotes the local integration of renewable energy. Specifically, compared to onshore distribution feeders, the operation of coastal feeders has the following unique features [3]:

1) Coastal feeders have a particular need for decarbonized energy, because fossil fuels are often not economically accessible to the onshore communities. This empowers coastal communities to take the lead in MHK resources.

2) Coastal feeders suffer some of the worst effects of extreme events caused by natural disasters. Therefore, they also have the greatest motivation to implement advanced grid service restoration techniques using offshore resources.

The operational resiliency of distribution systems around the world has been challenged by multiple major power outages in recent years. In 2017, Puerto Rico was hit by hurricane Irma, which left about 1 million customers without power. At the same time, Hurricane Harvey formed and brought around 300,000 power outages to customers in Texas and Louisiana. Because of their locations, coastal feeders are among the most affected areas during these events.

Normally, only portions of feeders would be affected by outages on distribution systems. The grid service can be restored by re-energizing isolated branches using adjacent feeders. However,under the impact of extreme events if no available neighboring power system or energization from upstream grids are present, the system would remain off-grid, and restoration would rely on local generation units, such as distributed generators (DGs). As power electronicsrelated techniques develop, power electronic-based interfaces are favored to integrate DGs with 
the external electric system because they can be controlled extensively and respond both quickly and dynamically.

The increasing penetration of inverter-interfaced DGs motivates the development of microgrids (MGs). An MG is defined as a group of interconnected loads and DGs within clearly defined electrical boundaries that act as a single controllable entity with respect to the grid [4]. MGs can improve the self-healing capabilities of distribution systems, because they can be operated autonomously in an islanded mode. Flexible solutions for the restoration of distribution systems can be enabled by MGs: an MG can pick up critical loads without energy from the main grid. After a power outage caused by an extreme event, the distribution system could be automatically divided into several independent MGs that operate autonomously [5].

Conventional MGs have clearly defined electric boundaries and interact with the external grid at a single point of common coupling (PCC). If multiple MGs coexist along the feeder, each MG would be treated as an independent entity that can operate either autonomously or connected to the main grid. However, as increasing numbers of MGs are created and integrated into the grid, proper coordination among the interconnected MGs can vastly improve reliability, resilience, and interoperable electrical service for conventional MG customers. The desire to link multiple MGs gives rise to a new concept of MGs, called networked MGs. Networked MGs, also called nested MGs, MG clusters, or aggregated MGs, refers to the interconnection of multiple adjacent MGs into one network [6]. Unlike conventional MGs, networked MGs could have multiple PCCs. The operating characteristics (load profile, power capacity, response dynamics, etc.) of each MG can be significantly different. The U.S. Department of Energy (DOE) envisions a networked MG structure as presented in Figure 1-1.

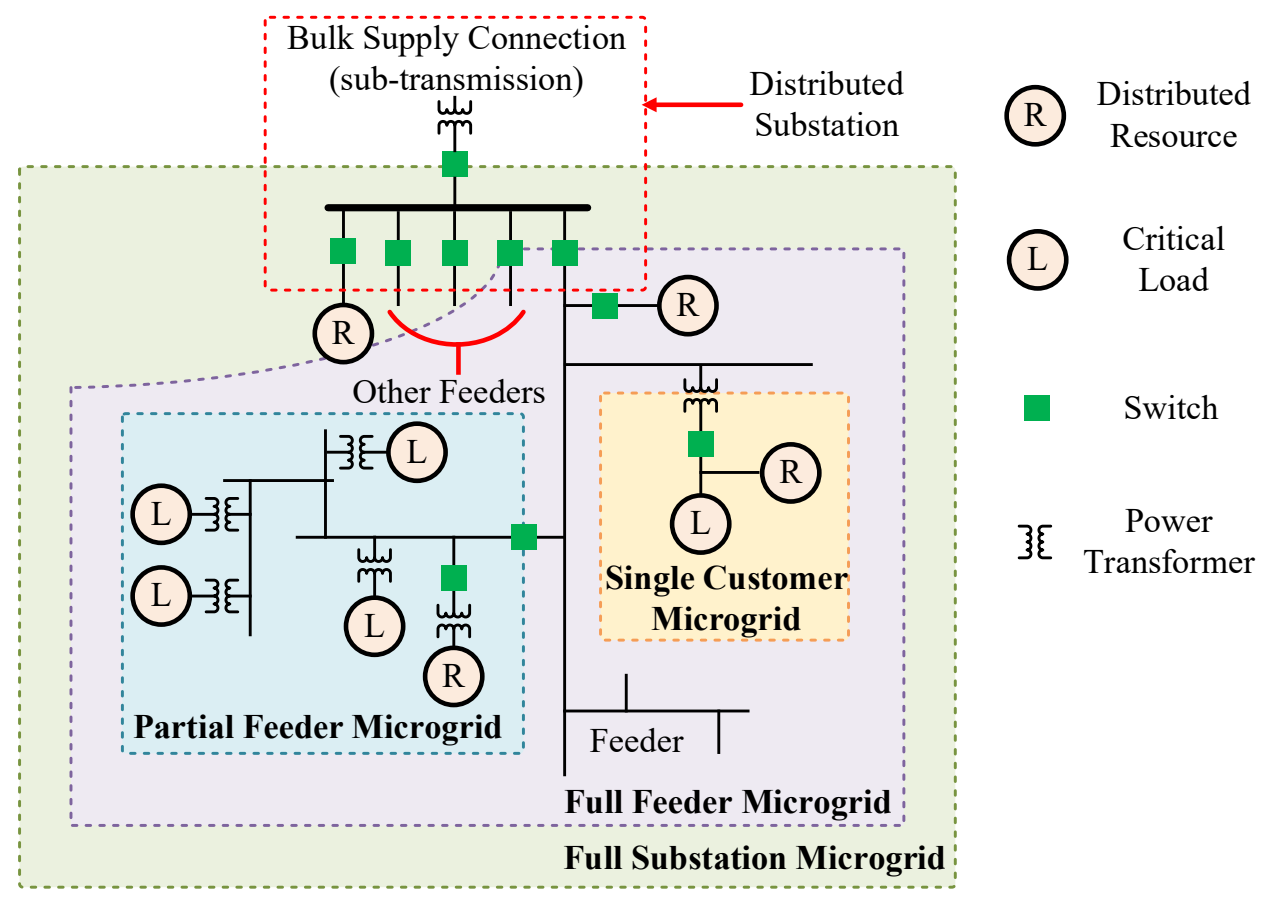

FIGURE 1-1 DOE Vision of Nested MG [6].

Coordination among networked MGs should be enabled under both grid-connected and islanded modes. Doing so could potentially improve system resiliency, operational efficiency, 
and economic benefits. Specifically, system operational resiliency could improve if areas with generation deficit are supported by other MGs are healthy. The cooperation among MGs enables them to use the most efficient generation methods to make sure the system run optimally. However, there are several operational challenges in networked MGs management:

- Connecting multiple MGs complicates system operation and coordination as the number of assets increases. Because the dynamics and controllability of the components within networked MGs would vary significantly, interactions among components with different operating characteristics should be carefully implemented.

- Managing multiple PCCs results in additional operational complexity (e.g., protection adjustment) that has not been extensively studied. Conventional modeling methods make the power systems operate with one slack bus and enforce unidirectional power flow. However, when several MGs are interconnected with multiple PCCs, the direction of power flow would be flexible. This feature enables flexible options for system operation, but also results in challenges that have not been studied in conventional power systems.

- Interactions among networked MGs become critical and require extra control effort as the networked MG framework becomes increasingly complex. As introduced, although each MG is interconnected with others, it is managed as an individual entity. However, the coordination among multiple interconnected MGs is critical for operational efficiency, stability, and resiliency.

The notion of dynamic MG has been recently studied as an advanced framework of networked MGs [7]. A dynamic MG is defined as an MG with flexible electric boundaries that could expand or shrink in response to requests from system operators. In other words, a dynamic MG possesses the fundamental features of a conventional MG (e.g., autonomous operation) with additional operational flexibility (e.g., dynamic electric boundaries). The differences between the conventional networked MGs and dynamic MGs are illustrated in Figure 1-2. Generally, conventional networked MGs operate under fixed topologies and are interconnected at a static PCC. the DGs are managed in a static group; while dynamic MGs have variable electric boundaries and interact at dynamic POIs. Therefore, the DGs would be actively re-grouped concerning the system operating topology. They can be described in detail as follows [8]:

- The electric boundaries of each static MG under the conventional networked MG framework are predefined, while the topology of a dynamic MG could vary. Specifically, the electric boundaries of dynamic MGs could vary in response to requests from system operators.

- Interconnections among conventional networked MGs are achieved through static breakers at the PCC. When the static breaker is open, the whole MG isolates from an external system and operates autonomously. However, a dynamic MG interacts with its neighboring MGs at multiple points of interconnection (POIs). As the topology of each dynamic MG varies, its POI would also vary accordingly. 

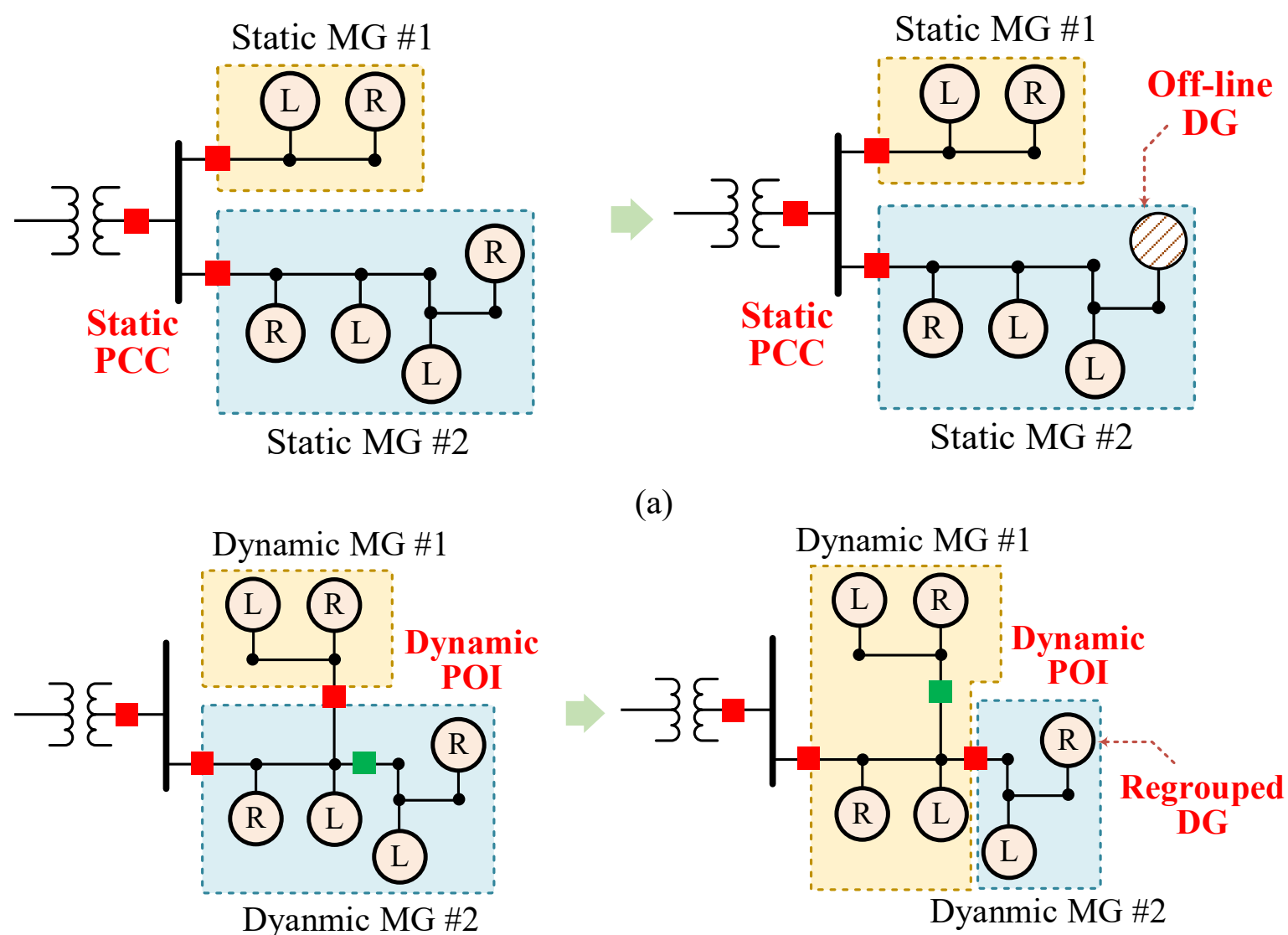

(a)

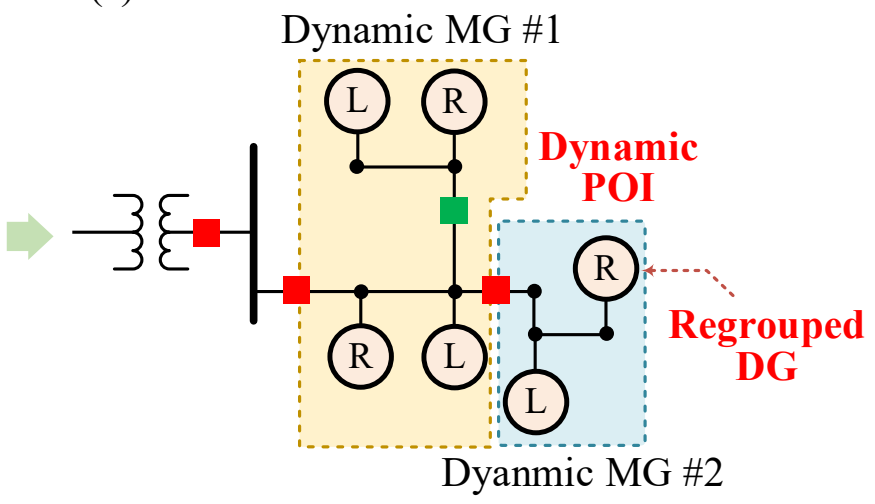

(b)

\section{FIGURE 1-2 Comparison between (a) Conventional Networked MGs and (b) Dynamic MGs.}

- In a conventional networked MG, the components (DGs, critical loads, etc.) within each static MG are managed in a predefined group. Coordination among components from different groups is usually not considered. However, because of the variation of the dynamic MGs' electric boundaries, the components that were previously isolated could be interconnected and efficiently managed as a group; this calls for the dynamic regrouping of components in response to the system's real-time topology.

As discussed, in addition to acting as autonomous resources in grid restoration, dynamic MGs could also participate in the reconfiguration of distribution system networks. Network reconfiguration has been widely adopted for operating distribution systems following a common power system contingency (e.g., power outages at electrical sections), where the topology of distribution feeders is altered by changing the open/closed states of the sectionalizing switches to assist grid operation (e.g., load pick-up, power flow mitigation over tie-lines). The static topology of conventional networked MGs would inevitably be represented as a hard constraint when solving the optimal reconfiguration plan, which could produce suboptimal solutions. However, dynamic MGs with varying electric boundaries could provide more flexible reconfiguration options for the system operators. 
However, conventional network reconfiguration techniques do not apply to grid restoration in the context of dynamic MGs. First, most existing works still rely heavily on energization from the utility, which might be unavailable during extreme events. Specifically, many works on network reconfiguration for grid restoration are developed with the prerequisite that there exists a robust and infinite bus in the system. However, as discussed, this is not always the case; the distribution system could be completely isolated from the substation, or the substation itself could even be at fault.

In addition, most existing works are formulated as an optimization problem that focuses on system steady-state performance; in other words, the system's steady-state operation after reconfiguration should not violate any operating limits [9]. However, conventional distribution systems are energized by synchronous generators with large inertia. Dynamic MGs would be mainly supported by inverter-based DGs with small inertia and thus become more vulnerable to transients. If the transients are out of bounds, inverter-based DGs will be forced to trip and the MG will be de-energized [10]. Because there is current continuity in inductive power lines, undesired transients will be introduced to the MG if there is non-zero power flow at the breaker that opens, or asynchronous voltage phasors at the breaker that closes [11].

Many works have promoted the use of dynamic MGs in distribution system network reconfiguration. In [10], the use of MGs in network reconfiguration is solved as an optimization problem that considers system dynamic operational constraints. After solving for the topology that allows the optimal system steady-state performance, the corresponding reconfiguration option is evaluated using dynamic simulation and would only be considered as feasible if the simulated operation status during reconfiguration does not violate the dynamic constraints. The reconfiguration options that might violate the system dynamic operational constraints would be effectively circumvented; however, such a prescreening approach would significantly increase the computation time and does not improve the feasibility of using MGs to reconfigure networks.

In [12], a control framework for synchronous generators is proposed that constantly regulates system operating frequency and voltage during network reconfiguration. During reconfiguration, the transients would be actively bounded. Less operation time is taken to achieve a successful MG network reconfiguration compared to the approach from [10] because no pre-simulation is required. However, the proposed control strategy is a passive solution to mitigate undesired transients, because it would not be energized unless the transients were well identified. The moment the breaker changed status, undesired transients - although boundedwould still be introduced to the system, and seamless network reconfiguration cannot be guaranteed while the system topology varies.

Deviation in system operations and transients could be essentially eliminated, as proved in [8], if the system is properly regulated. Specifically, the switch could seamlessly reclose if the voltage phasors from both sides were synchronous; on the other hand, switch could reopen seamlessly if its through current was minimized before the switch operation.

In [13], the authors proposed a control framework that integrates dynamic MGs into an existing distribution system. However, energization from neighboring substations plays a critical role in the proposed operation scheme, which limits the application of the developed work. 
In [14], the authors analyze the optimal demand response using dynamic MG reconfiguration. The proposed work finds the optimal electric boundaries of dynamic MGs under system contingencies. However, the work is still a steady-state problem where the undesired transients during reconfiguration are unbounded.

In [8], the authors propose a set of distributed secondary control strategies for dynamic MG operation that achieves constant system frequency/voltage regulation while achieving seamless topology variation during network reconfiguration.

For distribution systems that are unbalanced due to the common use of single- and two-phase laterals, [15] proposes a control strategy designed for grid-forming inverter interfaced DGs in the context of unbalanced dynamic MGs.

Finally, inverter-based distribution systems with dynamic MGs and reconfigurable cyber-physical systems (CPS) are discussed in [16]. Besides the varying topology under physical layer, the cyber network of the dynamic MGs could also be reconfigurable, which would further enable more feasible options for system operation with enhanced resiliency, while calls for additional coordination between the cyber and physical layers. 


\section{CHARACTERIZATION OF MHK RESOURCES}

\subsection{WAVE ENERGY}

Wave energy is fundamentally generated withthe continuous movement of waves that create a continually available and periodical energy source. Wave energy can be converted to electricity through a wave energy converter (WEC) whose motion drives a generator. A typical characteristic of wave generation is fluctuation. Compared to wind turbine generation, voltage variations caused by wave energy fluctuation are lower in frequency but higher in amplitude [17]. A sample power profile of a wave generator is shown in Figure 2-1 [18].

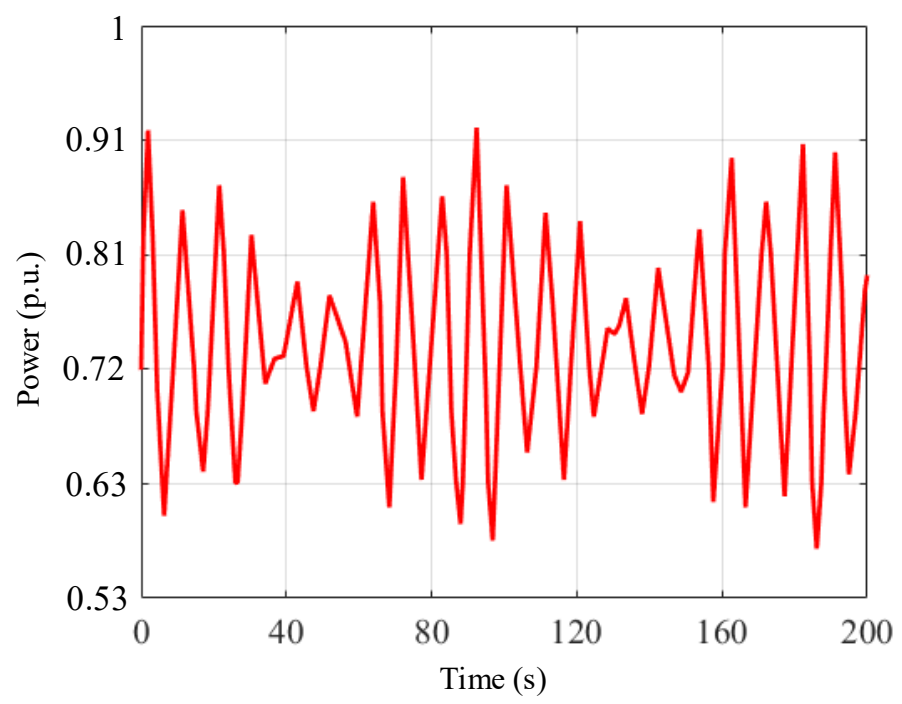

FIGURE 2-1 Sample Wave Generation Profile.

\subsection{TIDAL ENERGY}

Tidal energy is generated by the earth's rotation within its ocean envelope as shaped by the gravitational fields of the moon and sun [19]. Tidal energy can be converted to electricity through water height differences or kinetic energy created when alternating tides and geography induce water flows [20]. Tidal generation produces electrical power from roughly four tides each day. An important feature of tidal generation is that it is associated with the moon phases, as shown in Figure 2-2 [19], where a sample tidal current profile is converted to electrical power generated by tidal energy [21]. 


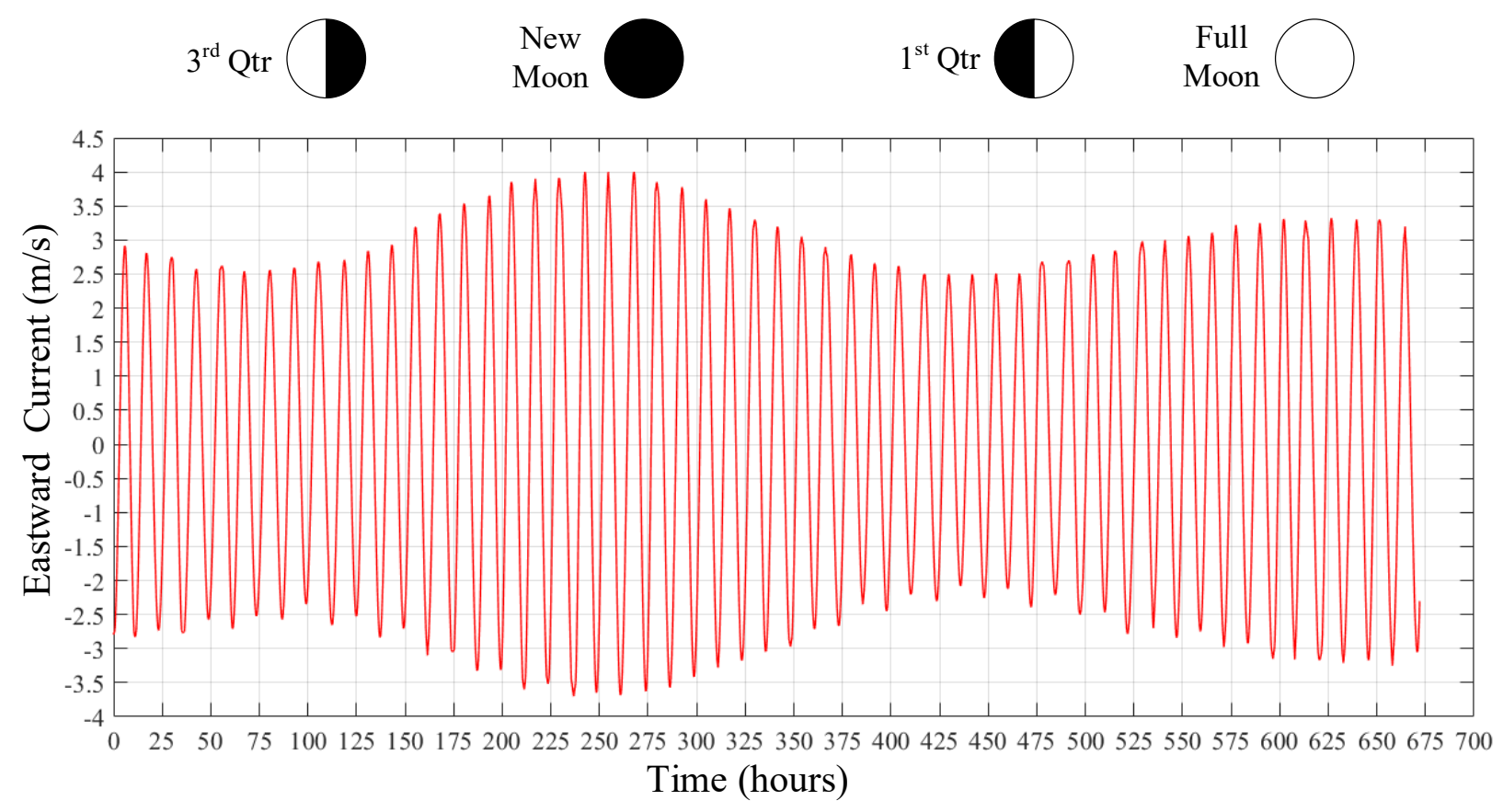

FIGURE 2-2 Sample Tidal Current Profile with Respect to Moon Phases. 


\section{COASTAL NETWORKED MG IMPLEMENTATION SURROUNDING MHK RESOURCES}

For a relatively long-term operation, it is possible to perform a series of restoration operations to help a coastal community integrated with MHK resources to recover from blackouts. These operations include: network reconfiguration enabled by forming networked MGs and altering their time-varying dynamic boundaries; real and reactive power generation dispatches for MHKs via solving optimization problems like economic dispatch problems; nodal voltage control through volt-var control; and other operations. These operations facilitate speedy load pick-up and ensure a resilient power supply with improved power quality.

The impacts of the aforementioned restoration operations on grid resilience are coupled and interdependent. For example, without a viable power distribution path that has sufficient capacity to connect an MHK to the rest of the network, the grid-supporting capability of the MHK will be significantly limited, regardless of how optimal the reactive and real power dispatch are. On the other hand, when dynamic MG boundaries are formed with an optimal structure, the grid supporting potentials of MHK resources can be better exploited to meet local and other demands in the system. Hence, their mutual impacts should be effectively characterized and taken into consideration when devising a restoration scheduling so that the joint enhancement effects on grid resilience can be maximized.

In particular, optimization techniques facilitate operating the coastal communities as dynamic MGs. We formulated a comprehensive optimization problem that co-models the restoration as well as the joint effects on system resilience. The solution of the problem is a time series sequence of restoration operations. It includes the control signals for the on/off status of the switching devices, the real and reactive power dispatches for MHK and other distributed energy resources, and nodal voltage for the energized components. These decision variables constitute the joint design of the dynamic MG configuration and operation strategies over time.

The problem is formulated as a mixed-integer programming problem. The solution algorithm is developed to co-optimize the configuration and voltage/power profiles in the system in order to maximize the energy supply (i.e., minimize the power outage time) at critical infrastructures.

Below, we briefly give an overview of the formula of the problem. The cost function of the problem is given by:

$$
\max \sum_{l \in L} \sum_{t \in T} \cdot \underbrace{\beta_{t}^{l}}_{\text {weight }} \cdot \underbrace{\Delta t \cdot \beta_{t}^{l}}_{\text {load demand }}
$$

where

- $L$ is the set of load indices,

- $T$ is the set of times that contain the time step index over the optimization horizon, 
- $\quad \beta_{t}^{l}$ is the weighting factor of load $l$ at time $t$,

- $\Delta t$ is the time duration of a time step,

- $\quad P_{t}^{l}$ is the power supply to load $l$ at time $t$.

The special features of the objective function are summarized as follows: (1) The cost function aims to maximize the energy supply (which is the product of the power suppy and the time duration) to coastal loads. Hence, the solution algorithm is guided to recover the power suppy as quickly as possible to guarantee a speedy restoration. (2) The service restoration tasks are ranked by priority for different loads. Each load demand is associated with a weighting factor, which indicates the urgency of picking up that load. Through this modeling technique, the loads are prioritized so that the optimization solvers can recognize the critical infrastructures; this allows them to meet these power supplies first to restore service more quickly to assets that are significant to the normal operations of a coastal community.

The problem is subject to a wide range of constraints to ensure that it applies to realistic scenarios. Such constraints are essential to guaranteeing that the designed restoration operations conform to various engineering standards and operational constraints. The constraints of the formulated optimization problem are summarized as follows:

- Dynamic MG configuration constraints-These model the power flow between and within the dynamic MGs as well as the varying boundaries between them. The power flow is modeled using DistFlow constraints that are amenable to computationally efficient solution algorithms for distribution power grids with a radial network. The dynamic boundaries are modeled by including binary decision variables for the on/off status of the switching devices of the power lines. These constraints define the connectivity and power flows of the entire network.

- MHK power generation constraints - These specify the variation ranges of different MHK resources. The parameters of the constraints vary over time. This flexible modeling approach can allow the the optimization problem to better reflect the MHK applications, where the MHK power output capability evolves over time.

- Energy storage system (ESS) constraints-ESSs can be co-sited with MHKs to smooth oscillations in outputs. Because ESSs usually have capacity limits, ESS operations need to account for the impacts of previous charging/discharging decisions on their future output capabilities. These dynamic constraints are also included in the optimization problems to facilitate the integration of ESSs.

- Voltage limit constraints-The voltage set-points of the system are co-designed with the network configuration and power output dispatches. The variation ranges of the voltage are included in the optimization problem to ensure that the optimization solutions satisfy the operational constraints. 
- Other constraints - In addition, we include a variety of other constraints, such as spinning reserve constraints and transformer and line capacity constraints. They jointly shape the decision variables to satisfy various requirements.

In particular, the optimization problem is tailored for a coastal community integrated with MHK resources. In light of the variability in the MHKs generation, the optimization seeks to restore the system service in a step-by-step sequence. The restored service in each step is designed in accordance with the available MHK resources.

We incorporate specific MHK constraints to ensure dispatching decisions to meet MHK operational requirements, such as output capability constraints and ramp rate constraints. These constraints also vary over time to represent the changing outputs of MHK resources. Because a coastal community is subject to a variety of sudden demand spikes caused by docking ships and related activities, we include capacity reserve constraints and constraints with respect to energy storage. Their inclusion can help to prepare coastal distribution grids for potential sudden demand increase.

It is worth mentioning that after the severe damages caused by a natural disaster, the coastal community could be disconnected from upstream grids and operate autonomously. To guarantee the system's operational resilience and to keep the critical loads constantly energized, all available resources from the coastal community should be used in coordination with each other.

As previously noted, different MHK resources have different operational characteristics, and the interactions among various MHK resources on the same feeder represent additional operatonal challenges compared to that on the inland feeders In addition, MHK resources alone could not act as reliable grid-forming units without help from dispatchable DGs (such as battery energy storage sources [BESSs], combined heat and power [CHP], etc.). To achieve full use of MHK resources with enhanced system operational resiliency, the coastal feeder could be operated in the context of dynamic MGs. In addition to the optimization formula, we implement a control framework for dynamic MG management using a decentralized control diagram. It was developed as a coupled CPS that achieves the requested system topology variation and coordinates various resources in the coastal feeder using a sparse communication network.

Both the physical and cyber networks of the developed framework are reconfigurable, which enables more flexible options for system operation during extreme conditions. We also propose a detailed secondary control strategy for operating coastal feeders: regulations over the system operating frequency and voltage are constantly enabled by the grid-forming DGs.

Proportional power-sharing among connected DGs in response to the feeder topology variation is achieved. The proposed control strategy helps stabilize the transient operation process and maintain the desired stability margin by achieving seamless system reconfiguration in response to the system operator's request. Finally, if the whole feeder is driven to a complete blackout due to extreme events, we propose a conceptual black start procedure in the context of the proposed dynamic MG operation framework. 
As discussed, there are in general two types of resources that exist in the coastal feeder, namely: (1) MHK resources with periodic and uncontrollable power generations and (2) dispatchable DGs with controllable power outputs. We assume that all the resources studied here are interfaced to the external grid through inverters [22]. A general control framework of inverter-based DG under hierarchical control framework is presented in Figure 3-1.

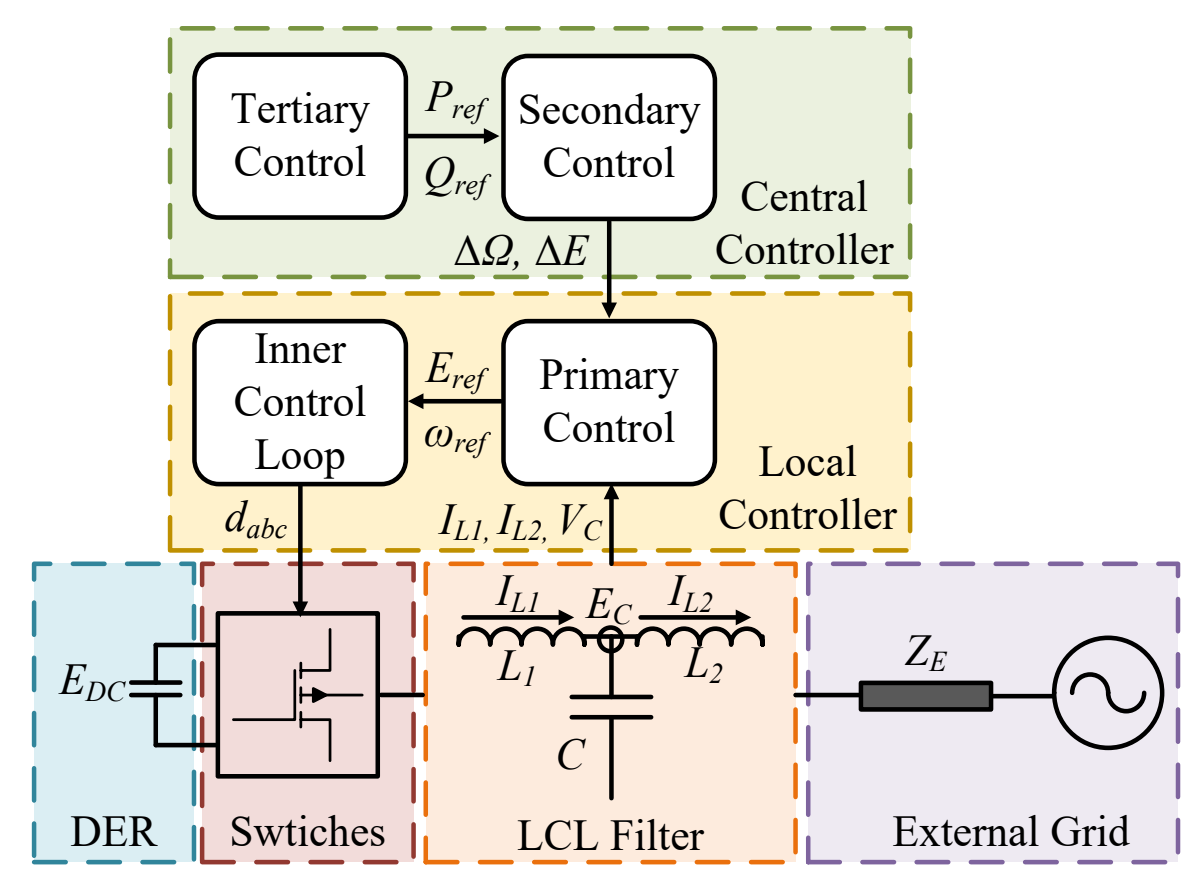

FIGURE 3-1 Conventional Structure of an Inverter-based DG [23].

An inverter-based DG can be treated as a controllable voltage source. There are in general two types of voltage source inverter (VSI): current-control mode (CCM) and voltagecontrol mode (VCM). VSI-CCM is designed for inverters that interface with non-dispatchable resources (e.g., MHK resources), where the DGs always operate in maximum power generation mode and inject grid-synchronized current to the external system. The terminal voltage phasor of VSI-CCM is controlled passively while its current output is actively regulated, which means VSI-CCM operates like a controllable current source. In contrast, VSI-VCM is designed for DG to operate as a grid-forming unit to stabilize the system's operating voltage and frequency. The terminal voltage of the VSI-VCM is actively regulated as a slack bus so that the whole system can reach power balance. VSI-VCM is usually designed for inverters that interface with dispatchable resources (e.g., BESS) because it can adjust its power outputs to seek the system's power balance point, which means VSI-VCM operates like an ideal controllable voltage source.

As previously discussed, the topology variation of a dynamic MG is fulfilled by changing the status of the switch. Generally speaking, a planned network reconfiguration in the power system starts with the system operator sending the reconfiguration request to the target switches that need to change status (either reclose or reopen). In this work, switches with intelligent 
electronic devices (IED) capability, called smart switches (SSWs), are used as the sectionalizers that are controlled by the system operator and define the electric boundaries of dynamic MGs. SSWs have the following advanced features compared to the conventional switches:

- When the system is operating normally, a conventional switch can only operate as a static break/connection point in the electric circuit, whose status is usually fixed at the planning stage (e.g., normally open [NO] switch or normally closed [NC]). However, an SSW could be equipped with advanced operational functionalities (e.g., remote controllability, peer communication) to serve the grid during operation. For example, SSW could actively participate in network reconfiguration by changing its status in real time as requested, so that the system can operate more efficiently.

- When the system is operating abnormally, a conventional switch usually acts as a protection device that follows the implanted operation scheme using local measurements (e.g., over current protect), which can limit the operational flexibility of inverter-based dynamic MGs and easily drive the system into a blackout. An SSW, on the other hand, can actively participate in sectionalizing the feeder into subsections following a remote command [5], so that each subsection can operate autonomously and the critical loads can be constantly energized.

Finally, note that a number of SSWs have already been commercialized and implemented in the field. For example, the IntelliRupter ${ }^{\circledR}$ from S\&C Electric Company has been implemented in Alberta, Canada, to save more than 30 million customer minutes of power interruption [24].

In this work, we propose a control framework for coastal feeder operation in the context of dynamic MGs. The DGs, including MHK resources and dispatchable resources, are all inverter-interfaced and selected as the primary resources when the coastal feeder operates autonomously. SSWs are used to sectionalize the whole feeder into predefined subsections. Each subsection is called a minimum MG (min-MG). Each min-MG is defined as the smallest set of DGs and SSWs that can support local critical loads by forming an autonomous MG. Specifically, as required by Institute of Electrical and Electronics Engineers (IEEE) Standard 1547, the total installed generation capacity of each min-MG should exceed the total critical loads within [4]. In addition, because MHK resources alone cannot energize the feeder as a grid-forming unit, each min-MG must contain at least one DG under VSI-VCM. The proposed dynamic MG control framework for coastal feeder operation is presented schematically in Figure 3-2.

As shown in Figure 3-2, the whole feeder is sectioned into adjacent min-MGs. The electric boundaries of each min-MG are determined by the position of SSWs. The adjacent min-MGs could be interconnected by the closed SSW they share and operate as a whole, and the min-MGs that are isolated from the external grid by the open SSW could also operate independently. Specifically, each min-MG is designed to be building block of a dynamic MG. The whole feeder would essentially operate in the context of dynamic MGs, where each dynamic MG consists of one or more min-MGs. The topology variation of each dynamic MG is fulfilled by turning the corresponding SSWs on and off; the network could be reconfigured per the system operator's request, because the SSWs can be controlled remotely. The following rules should be 
applied when choosing a location for the SSW: (1) Each min-MG has at least one POI that interconnects to the

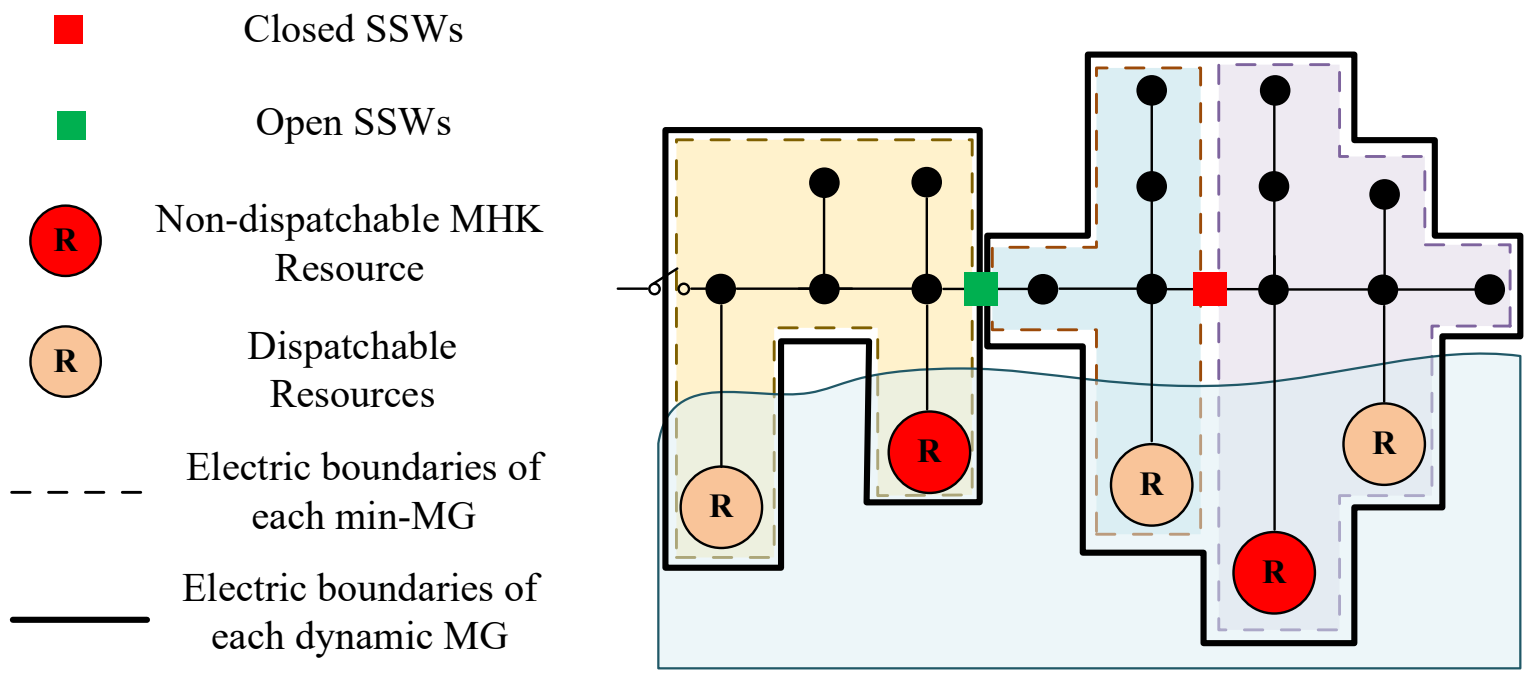

FIGURE 3-2 Dynamic MG Control Framework for Coastal Feeder.

rest of the grid via SSW. This ensures the controllability of the system operator over the system's operating topology. (2) Two adjacent min-MGs share only one SSW, which can be can be easily achieved in a radial distribution system. This simplifies the interactions between the two minMGs without losing generality.

As discussed, to further use the operational flexibility of dynamic MGs, both its cyber and physical networks should be reconfigurable. We assume that the DGs and SSWs in the proposed control framework are managed as an intelligent agent; each agent could exchange selected information peer-to-peer through bidirectional communication links. In [16], the authors describe ways in which cyber- and physical networks are both independent of and interdependent on the operation of dynamic MGs. The operational characteristics of the cybernetwork and physical network during reconfiguration in the context of dynamic MG operation are inherently different, and the connectivity of these networks are not necessarily identical:

- The physical network's topology under normal conditions could only be varied by changing the status of the corresponding SSW. The breaking and connection points are fixed by the position of SSW, and the possible system operating topology could be traversed by the on and off combinations of all the SSWs. However, there could be various possible topologies for the cyber network, and its topology variation is only constrained by the availability of cyber components, such as network switches and Ethernet cables. 
- The physical connectivity among neighboring agents is strictly determined by the feeder's geometric structure. Specifically, each DG can only be physically connected with its neighboring DGs through transmission lines and closed SSWs, and will be disconnected by any open SSW in between. However, the connectivity of cyber networks does not have these types of constraints. Specifically, agents could be connected in the same cyber network for information exchange even if they are isolated physically. The system operator could actively reconfigure the communication links among agents to enhance the system's operational resiliency.

In addition to the aforementioned independence of cyber and physical networks, their interdependency could be described by the fact that the resilient operation of the physical network depends on a properly functional cyber network. Coordination among DGs relies on information exchange; the physical network is reconfigured by SSW operation, which is remotely controlled by the system operator. However, sufficient connectivity should always remain in the cyber network to guarantee the resilient operation of the physical network [25]. Specifically, under the distributed control framework, all the DGs that are physically connected should also be connected within the same cyber network to ensure sufficient distributed controllability [16].

As discussed, proper regulation for dynamic MGs provides more feasible reconfiguration solutions with enhanced system robustness. Three-level hierarchical control has been widely adopted for MG operation, namely primary, secondary, and tertiary control [22]. Primary control is based on the droop control to stabilize system operating frequency and voltage. Droop control is usually adopted at the primary level, which usually results in steady-state deviations on the system's operational states. Secondary control is introduced to ensure operational states are regulated as desired (e.g., as rated). Tertiary control manages the power flow between the MG and the external electrical distribution system so that the system operates economically.

Regulation for dynamic MG operational resiliency falls under secondary control. Compared to centralized control schemes, decentralized or distributed control strategies have been favored in recent research on MG secondary control [26]. Distributed secondary control strategies have been widely employed to coordinate operations among DGs. However, the secondary control strategy designed for dynamic MG operation calls for extra control efforts compared to the approaches for conventional MGs:

- Conventional secondary control is designed for MGs with static boundaries and PCC. Conventional secondary control objectives mainly focus on improving the system performance under static topology [27], such as frequency/voltage regulation and DG power-sharing. However, dynamic MGs operate with changing topology and dynamic POIs. In addition to constant regulations of system operating frequency and voltage, secondary control for dynamic MGs needs to coordinate the interconnection of MGs at changing POIs to ensure seamless dynamic MGs topology variation. Specifically, the through current needs to be minimized before the SSW opens, and the voltage phasors on both sides of the SSW need to be synchronized before the SSW closes. 
- Each DG in the static MG will be assigned to a given MG with predetermined boundaries; the interconnected DGs would coordinate at the secondary control level and form a static group. This approach fails when the system is reconfigured; DGs that previously belonged to different groups are interconnected and should be managed under a new group because they are no longer isolated. As shown in Figure $1-2$, the DG groups need to be managed dynamically in response to real-time variations in system topology. DGs that are interconnected after the reconfiguration should achieve proportional power-sharing through regrouping to prevent improper power flow within the reconfigured dynamic MGs.

In support of the proposed dynamic MGs operational framework, a set of the distributed controller is designed for the dispatchable DGs, while all the MHK resources operate in maximum power output mode and are treated as reversed power consumption in the subsequent analysis. In this work, we focus on providing secondary level regulation for the coastal feeder in the context of dynamic MGs operation in islanded mode. In addition to constant system frequency and/or voltage regulation, the proposed controllers have three modes of operation:

- Type I is designed for dynamic MG operation when no network reconfiguration is requested. The feeder operates under static topology. Proportional power-sharing among DGs that are physically connected is achieved.

- Type II is designed for dynamic MG network reconfiguration when a system operator asks a closed SSW to open. To achieve seamless system topology transition, power flows through the target SSW are eliminated before the target SSW changes status.

- Type III is designed for dynamic MG network reconfiguration when a system operator asks an open SSW to close. To achieve seamless system topology transition, voltage phasors on both sides of the target SSW are synchronized.

To avoid introducing contradicting regulation efforts to the system at the same time, only one operational mode is activated at a time on each DG. The operational mode of the $i^{\text {th }}$ DG is indicated by a pair of binary variables, $\eta_{i}, \lambda_{i}$ :

- $\eta_{i}=0, \lambda_{i}=0$ indicates the energization of Type I mode. The DG provides regulation in the context of dynamic MG operation with static topology.

- $\eta_{i}=1, \lambda_{i}=0$ indicates the energization of Type II mode. The DG supports dynamic MG topology variation through seamless SSW reopening.

- $\eta_{i}=0, \lambda_{i}=1$ indicates the energization of Type III mode. The DG supports dynamic MG topology variation through seamless SSW recloseing.

Note that the operational modes of each DG should be determined by the system operator and are not necessarily identical when network reconfiguration is requested. For example, when a system operator asks a target SSW to open, the DGs that have electric interconnections through 
the target SSW should switch from Type I mode to Type II mode, while the DGs that are not physically connected to the target SSW would remain in Type I mode.

The DG operating frequency/active power controllers are presented. For the $i$ th DG:

$$
\begin{gathered}
\omega_{i}=\omega^{*}-m_{i} P_{i}+\Omega_{M_{1}}+\Omega_{M_{2}}+\Omega_{M_{3}} \\
\dot{\Omega}_{M_{1}}=-k_{f}\left(\omega_{i}-\omega^{*}\right)-\left(1-\lambda_{i}\right) k_{p} \sum a_{i j}\left(P_{i}^{\prime}-P_{j}^{\prime}\right) \\
\dot{\Omega}_{M_{2}}=-\lambda_{i} k_{p}\left(P_{S S W}^{\prime}-0\right) \\
\dot{\Omega}_{M_{3}}=-\eta_{i} k_{\theta}\left(\theta_{S S W}-0\right)
\end{gathered}
$$

where $\omega_{i}$ and $\omega^{*}$ are the DG operation and rated frequency, respectively; $m_{i}$ is the P-f droop gain; $P_{i}$ and $P_{i}{ }^{\prime}$ are the DG active power output in Watt and per-unit, respectively; $\Omega_{M 1}, \Omega_{M 2}$, and $\Omega_{M 3}$ are the designed control variables that fulfill the control objectives at each mode of operation:

- $\Omega_{M 1}$ is designed for DG operation in Type I mode, where $k_{f}$ and $k_{p}$ are the designed control gains; $a_{i j}$ indicates the data exchange between the $i$ th and $j$ th $\mathrm{DG}$, it is valid when $a_{i j}=1$ and invalid when $a_{i j}=0$;

- $\Omega_{M 2}$ is designed for DG operation at Type II mode, where $P_{S S W}{ }^{\prime}$ is the per-unit active power flow at the target SSW that awaits reopening;

- $\Omega_{M 3}$ is designed for DG operation in Type III mode, where $k_{\theta}$ is the designed control gain; $\theta_{S S W}$ is the phase mismatch at the target SSW that awaits reclosing;

As shown in equations (3.1) through (3.4), different control variables are activated under corresponding modes of operation:

- In Type I (as $\lambda_{i}=0$ and $\eta_{i}=0$ ), $\omega_{i}=\omega^{*}$ and $P_{i}{ }^{\prime}=P_{j}{ }^{\prime}$ as the controller converges, meaning that the DG operating frequency, and thus the system operating frequency, is regulated as rated and proportional DG active power-sharing has been achieved.

- In Type II (as $\lambda_{i}=1$ and $\left.\eta_{i}=0\right), \omega_{i}=\omega^{*}$ and $P_{S S W^{\prime}}=0$ as the controller converges, meaning that the system operating frequency is regulated as rated and the active power flow at the target SSW is minimized for seamless re-open.

- In Type III (as $\lambda_{i}=0$ and $\eta_{i}=1$ ), $\omega_{i}=\omega^{*}, P_{i}{ }^{\prime}=P_{j}{ }^{\prime}$, and $\theta_{S S W}=0$ as the controller converges, meaning that the system operating frequency is regulated as rated, proportional DG active power-sharing is achieved, and the phase mismatch at the target SSW is minimized for seamless reclosing. 
The DG operating voltage/reactive power controllers are presented. For the $i$ th DG:

$$
\begin{gathered}
E_{i}=E^{*}-n_{i} Q_{i}+\Pi_{M_{1}}+\Pi_{M_{2}}+\Pi_{M_{3}} \\
\dot{\Pi}_{M_{1}}=-\left(1-\eta_{i}\right) k_{V}\left(\phi_{i}-E^{*}\right)-\left(1-\lambda_{i}\right) k_{q} \sum a_{i j}\left(Q_{i}^{\prime}-Q_{j}^{\prime}\right) \\
\dot{\Pi}_{M_{2}}=-\lambda_{i} k_{q}\left(Q_{S S W}^{\prime}-0\right) \\
\dot{\Pi}_{M_{3}}=-\eta_{i} k_{V}\left(E_{S S W}-E^{*}\right)
\end{gathered}
$$

where $E_{i}$ and $E^{*}$ are the DG operation and rated voltage, respectively; $n_{i}$ is the Q-V droop gain; $Q_{i}$ and $Q_{i}{ }^{\prime}$ are the DG reactive power output in VAR and per-unit, respectively; $\Pi_{M 1}, \Pi_{M 2}$, and $\Pi_{M 3}$ are the designed control variables for each mode of operation:

- $\Pi_{M 1}$ is designed for DG operation at mode $M_{1}$, where $k_{v}$ and $k_{q}$ are the designed control gains; $\phi_{i}$ represents the average DG voltage observed at the $i$ th DG, which can be achieved using dynamic consensus-based average observer [28]:

$$
\phi_{i}=E_{i}-\int a_{i j}\left(\phi_{i}-\phi_{j}\right) d t
$$

- $\Pi_{M 2}$ is designed for DG operation at mode $M_{2}$, where $Q_{S S W^{\prime}}$ is the per-unit reactive power flow at the target SSW that awaits reopening.

- $\Pi_{M 3}$ is designed for DG operation at mode $M_{3}$, where $k_{V}$ is the designed control gain; $E_{S S W}$ is the voltage measured at the target SSW that awaits reclosing.

As shown in equations (3.5) through (3.9), different control variables are activated under corresponding modes of operation:

- In Type I (as $\lambda_{i}=0$ and $\left.\eta_{i}=0\right), \phi_{i}=E^{*}$ and $Q_{i}{ }^{\prime}=Q_{j}{ }^{\prime}$ as the controller converges, meaning that the average DG operating voltage is regulated as rated, and thus the system operating voltage is regulated at an acceptable range; proportional DG reactive power sharing is achieved.

- In Type II (as $\lambda_{i}=1$ and $\eta_{i}=0$ ), $\phi_{i}=E^{*}$ and $Q_{S S W^{\prime}}=0$ as the controller converges, meaning that the system operating voltage is regulated at an acceptable range and the reactive power flow at the target SSW is minimized for seamless reopening.

- In Type III (as $\lambda_{i}=0$ and $\eta_{i}=1$ ), $Q_{i}{ }^{\prime}=Q_{j}{ }^{\prime}$ and $E_{S S W}=E^{*}$ as the controller converges, meaning that proportional DG reactive power sharing is achieved and the voltage at the target SSW is regulated as rated. Note that by applying the same regulation to the DGs on both sides of the SSW, voltage mismatch at the target SSW is minimized for seamless reclosing.

We analyzed the stability of the proposed controller. The following lemmas are used for subsequent stability analysis: 
- Lemma 1 [29]: If $\mathbf{M}$ is positive-definite and $\mathbf{N}$ is a positive-definite scalar, then $\mathbf{M N}=\mathbf{N M}$ is positive-definite and $\mathbf{M}+\mathbf{N}$ is positive-definite.

- Lemma 2 [27]: Characteristics polynomial in the following forms: $\operatorname{det}\left(s^{2} \mathbf{I}+s \mathbf{A}_{1}+\mathbf{A}_{2}\right)=0$ has all its roots satisfy $\operatorname{Re}(s)<0$ if both $\mathbf{A}_{1}$ and $\mathbf{A}_{2}$ are positive-definite.

- Lemma 3 [8]: $\mathbf{M}=\operatorname{diag}\left(M_{i}\right)$ is a positive-definite scalar and $\mathbf{L}$ is a Laplacian matrix of a connected graph. $\mathbf{L}+r[\mathbf{1}]_{n} \mathbf{M}$ is positive-definite when $r$ is a positive constant.

The system operating status of the inverter-interfaced DGs with grid-forming capability is modeled as follows:

$$
\begin{gathered}
\dot{\delta}_{i}=-m_{i} \frac{E^{\prime} E^{*}}{X_{i}} \delta_{i}+\Omega_{M_{i}} \\
\omega_{v}^{-1} \dot{E}_{i}=-E_{i}-n_{i} \frac{E^{*}}{X_{i}} \cos \delta^{\prime} E_{i}+\Pi_{M_{i}}
\end{gathered}
$$

where the delay in adjusting DG output frequency is ignored, while the delay in adjusting DG output voltage magnitude is modeled as a first-order low-pass filter [27]. $X_{i}$ is the equivalent reactance between the $i$ th inverter and PCC. Denote $M_{i}=E^{\prime} E^{*} / X$ and $N_{i}=\left(E^{*} / X_{i}\right) \cos \delta^{\prime}$ as constants. Finally, it is denoted that $\mathbf{M}=\operatorname{diag}\left(M_{i}\right), \mathbf{N}=\operatorname{diag}\left(N_{i}\right), \mathbf{m}=\operatorname{diag}\left(m_{i}\right)$, and $\mathbf{n}=\operatorname{diag}\left(n_{i}\right)$. Denote $[\mathbf{1}]_{\mathbf{M}, \mathbf{N}}$ as an M-by-N all-ones matrix.

The linearized system modeling with the proposed frequency/active power regulations is presented in matrix form as:

$$
\dot{\mathbf{X}}_{\delta}=\mathbf{W}_{\delta} \mathbf{X}_{\delta}
$$

where $\mathbf{X}_{\delta}$ is system states and $\mathbf{W}_{\delta}$ is the corresponding system matrix.

When Type I mode is activated and no reconfiguration is requested, the system matrix is derived as:

$$
\mathbf{W}_{\delta}=\left(\begin{array}{cc}
-\mathbf{m} \mathbf{M} & \mathbf{I} \\
k_{f} \mathbf{m} \mathbf{M}-k_{p} \mathbf{H}_{\mathrm{I}} \mathbf{M} & -k_{f} \mathbf{I}
\end{array}\right) .
$$


Matrix $\mathbf{H}_{I}$ is the Laplacian matrix that describes direct communication links among DGs. The characteristic polynomials of $\mathbf{W}_{\delta}$ is simplified using Schur complement:

$$
\operatorname{det}\left(s \mathbf{I}-\mathbf{W}_{\delta}\right)=\operatorname{det}\left(s \mathbf{I}+k_{f} \mathbf{I}\right) \operatorname{det}\left[s^{2} \mathbf{I}+s(\mathbf{m} \mathbf{M})+k_{p} \mathbf{H}_{\mathrm{I}} \mathbf{M}\right]=0 .
$$

We observe that $\operatorname{det}\left(s \mathbf{I}+k_{f} \mathbf{I}\right)=0$ satisfies $\operatorname{Re}(s)<0$ for all its roots. Referring to Lemma 1, when $M_{i} \approx M_{j}$, the matrix $\mathbf{H}_{\mathrm{I}} \mathbf{M}$ is positive-definite. Then, referring to Lemma 2, we conclude that all the roots of $\operatorname{det}\left[s^{2} \mathbf{I}+s(\mathbf{m} \mathbf{M})+k_{p} \mathbf{H}_{\mathrm{I}} \mathbf{M}\right]=0$ satisfy $\operatorname{Re}(s)<0$. Thus, in this scenario, the system is exponentially stable if $M_{i} \approx M_{j}$.

When Type II mode is activated and the target SSW is requested to open, the system matrix becomes:

$$
\mathbf{W}_{\delta}=\left(\begin{array}{cc}
-\mathbf{m} \mathbf{M} & \mathbf{I} \\
k_{f} \mathbf{m} \mathbf{M}-k_{p} \mathbf{H}_{\mathrm{II}} \mathbf{M} & -k_{f} \mathbf{I}
\end{array}\right)
$$

where $\mathbf{H}_{\mathrm{II}}=\mathbf{H}_{\mathrm{I}}+\left(k_{\Delta P} / k_{P}\right) \mathbf{D}_{\mathbf{o}^{\prime}}$. Assume there are $n_{L}$ and $n_{R}$ DGs on each side of the target SSW. In this case, $\mathbf{H}^{\prime}$ is guaranteed to be $\operatorname{diag}\left(\mathbf{H}^{\prime}{ }_{\mathbf{n L}, \mathbf{n L}}, \mathbf{H}^{\prime} \mathbf{n R}, \mathbf{n R}\right)$. The matrix $\mathbf{D o}^{\prime}$ describes the through power at the target SSW from (equation 3.3) with proper direction. Referring to the autonomous system power balance between generation and consumption, we derive that

$$
\left|\Delta P_{S S W}\right|=\left|2 \sum_{i \in N_{l}} P_{i}-P_{L}\right|=\left|2 \sum_{j \in N_{R}} P_{j}-P_{L}\right|
$$

where $P_{L}$ is the system total power consumption. We derived that $\mathbf{D}_{\mathbf{O}^{\prime}}=2 \operatorname{diag}\left([\mathbf{1}]_{\mathbf{N}_{L}, \mathbf{N}_{\mathbf{L}}},[\mathbf{1}]_{\mathbf{N}_{\mathbf{R}}, \mathbf{N}_{\mathbf{R}}}\right)$. Referring to Lemma 3, $\mathbf{H}_{\text {II }}$ is positive-definite. Similar to the previous case, the system is exponentially stable if $M_{i} \approx M_{j}$.

When Type III mode is activated as the target SSW is requested to close, the system matrix becomes:

$$
\mathbf{W}_{\delta}=\left(\begin{array}{cc}
-\mathbf{m} \mathbf{M} & \mathbf{I} \\
k_{f} \mathbf{m} \mathbf{M}-k_{p} \mathbf{H}_{\mathrm{III}} \mathbf{M} & -k_{f} \mathbf{I}
\end{array}\right)
$$

where $\mathbf{H}_{\mathrm{III}}=\mathbf{H}_{\mathrm{I}}+\left(k_{\theta} / k_{P}\right) \mathbf{D}_{\mathbf{C}^{\prime}} \mathbf{M}^{-1}$. The matrix $\mathbf{D}_{\mathbf{C}^{\prime}}$ describes phase mismatch at the target SSW from equation (3.4) compared to the common reference. Referring to Millman's theorem, voltage phasor measured by SSW could be expressed in a complex form: $\left|V_{S S W}\right|\left(1+j \theta_{S S W}\right)=\Sigma c_{i} E_{i}(1+$ $\left.j \delta_{i}\right)$ where $c_{i}=X_{p} / X_{i}$ and $\left(X_{p}\right)^{-1}=\Sigma\left(X_{i}\right)^{-1}$. It is derived that $\mathbf{D}_{\mathbf{C}^{\prime}}=[\mathbf{1}]_{\mathbf{n}, \mathbf{n}}$ with $\mathbf{D}=\operatorname{diag}\left(\mathrm{E}^{\prime} /\left|V_{X}\right| c_{i}\right)$. When $M_{i} \approx M_{j}, \mathbf{M}^{\mathbf{1}}$ is a diagonal positive-definite scalar. Referring to Lemma $3, \mathbf{H}_{\text {III }}$ is positivedefinite. Similar to the previous case, the system is exponentially stable if $M_{i} \approx M_{j}$.

The linearized system modeling with the proposed voltage/reactive power regulations is presented in matrix form as: 


$$
\dot{\mathbf{X}}_{E}=\mathbf{W}_{E} \mathbf{X}_{E}
$$

where $\mathbf{X}_{E}$ is system states, $\mathbf{W}_{E}$ is the corresponding system matrix.

When Type I mode is activated and no reconfiguration is requested, the system matrix is derived as:

$$
\mathbf{W}_{E}=\left(\begin{array}{cc}
-\mathbf{I}-\mathbf{n} \mathbf{N} & \mathbf{I} \\
-k_{E} \mathbf{T}-k_{Q} \mathbf{K}_{\mathrm{I}} \mathbf{N} & \mathbf{0}
\end{array}\right)
$$

where $\mathbf{T}=n^{-1}[\mathbf{1}]_{\mathbf{n}, \mathbf{n}}$ describes the average DG voltage regulation from equation (3.9) and $\mathbf{K}_{\mathrm{I}}=\mathbf{H}_{\mathrm{I}}+\mathbf{I}$. Set $k_{E}=0$ and the characteristic polynomials of $\mathbf{W}_{E}$ is expressed as:

$$
\operatorname{det}\left(s \mathbf{I}-\mathbf{W}_{\mathbf{E}}\right)=\operatorname{det}(s \mathbf{I}+\mathbf{I}+\mathbf{n N}) \operatorname{det}\left[s^{2} \mathbf{I}+s(\mathbf{I}+\mathbf{n N})+k_{Q} \mathbf{K}_{\mathbf{I}} \mathbf{N}\right]=0 .
$$

The term $\operatorname{det}(s \mathbf{I}+\mathbf{I}+\mathbf{n N})=0$ satisfied $\operatorname{Re}(s)<0$ for all its roots. Referring to Lemma 1, the matrix $\mathbf{I}+\mathbf{n N}$ is positive-definite. When $N_{i} \approx N_{j}$, the matrix $\mathbf{K}_{\mathbf{I}} \mathbf{N}$ is positive-definite. Then referring to Lemma 2, we conclude that when $k_{E}=0$ and $N_{i} \approx N_{j}$, all the roots of the characteristic polynomials satisfy $\operatorname{Re}(s)<0$. Because eigenvalues are a continuous function of matrix parameters, the previous conclusion still holds if $k_{E}>0$ is sufficiently small and the system is exponentially stable.

When Type II mode is activated as the target SSW is requested to open, the system matrix becomes:

$$
\mathbf{W}_{E}=\left(\begin{array}{cc}
-\mathbf{I}-\mathbf{n N} & \mathbf{I} \\
-k_{E} \mathbf{T}-k_{Q} \mathbf{K}_{\mathrm{II}} \mathbf{N} & \mathbf{0}
\end{array}\right)
$$

where

$$
\mathbf{K}_{\mathrm{II}}=\mathbf{K}_{\mathrm{I}}+\frac{k_{\Delta Q}}{k_{Q}} \mathbf{J}_{\mathbf{O}^{\prime}}
$$

The matrix $\mathbf{J o}^{\prime}=2 \operatorname{diag}\left([\mathbf{1}]_{\mathrm{NL}}, \mathbf{N L},[\mathbf{1}]_{\mathrm{NR}}, \mathbf{N R}\right)$ describes the through power at the target SSW from equation (3.7) with the correct direction. Referring to Lemma 3, the matrix $\mathbf{K}_{\text {II }}$ is positive-definite. Thus, $\mathbf{K}_{\text {II }} \mathbf{N}$ is positive-definite referring to Lemma 1 . Similar to the previous case, the system is exponentially stable if $k_{E}>0$ is sufficiently small and $N_{i} \approx N_{j}$. 
When Type III mode is activated as the target SSW is requested to close, the system matrix becomes:

$$
\mathbf{W}_{E}=\left(\begin{array}{cc}
-\mathbf{I}-\mathbf{n} \mathbf{N} & \mathbf{I} \\
-k_{Q} \mathbf{K}_{\mathrm{III}} \mathbf{N} & \mathbf{0}
\end{array}\right)
$$

where

$$
\mathbf{K}_{\mathrm{III}}=\mathbf{K}_{\mathrm{I}}+\frac{k_{\Delta V}}{k_{Q}} \mathbf{J}_{\mathrm{C}^{\prime}} \mathbf{N}^{-1}
$$

Matrix $\mathbf{J}_{\mathbf{C}^{\prime}}$ is a matrix that describes the voltage magnitude mismatch at the target SSW from equation (3.8). Note that in this case the term $k_{E} \mathbf{T}$ is not effective because average DG voltage regulation is disabled. As discussed, the voltage at target SSW can be expressed using Millman's theorem, and we derive that $\mathbf{J}_{\mathbf{C}^{\prime}}=[\mathbf{1}]_{\mathbf{n}, \mathbf{n}} \mathbf{C}$ with $\mathbf{C}=\operatorname{diag}\left(c_{i}\right)$. When $N_{i} \approx N_{j}, \mathbf{N}^{\mathbf{1}}$ is a diagonal positive-definite scalar. Referring to Lemma $3, \mathbf{J}_{\mathbf{C}}$ is positive-definite. Similar to the previous case, the system is exponentially stable if $N_{i} \approx N_{j}$. The value of $k_{E}$ does not affect the system stability in this scenario.

Finally, we conceptually introduce the use of the proposed dynamic MGs in implementing black start to a blackout coastal feeder. As discussed, due to their geography, coastal feeders would be among the most affected areas during extreme events. If the system is forced to blackout, it is critical to re-energize the feeder and restore grid service quickly and reliably.

Self-organized inverter-interfaced DGs have black start capability; they can start without offsite power and regulate system operating voltage and frequency [30]. Compared to the conventional black start units (e.g., diesel generators), inverter-interfaced DGs have a simplified black start sequence and greater controllability. However, because synchronous generators have different operating characteristics from inverter-based DGs, controllers designed for typical black start units do not apply to the inverter-based ones.

As required in IEEE Standard 2030.7 [31], MG control systems dispatch MG assets to perform black start in MG without a grid connection. Instead of remaining under outage, the blackout feeder could be re-energized using black start DGs by forming multiple autonomous MGs [32]. Generally, power system restoration is complicated and has many operating challenges. To implement a successful black start with enhanced operational resiliency, the restoration should follow predefined procedures.

To fully use the installed capacity during restoration, all available resources should pick up as much load as possible. However, MHK resources have considerable but non-dispatchable power outputs, which make the restoration of coastal feeders more challenging compared to the inland ones. On the one hand, the participation of MHK resources during the restoration could provide sufficient power to pick up more customers; on the other hand, use of MHK resources 
should be carefully implemented without destabilizing the feeder. Specifically, during the restoration, due to the randomness of both the generation of MHK resources and the loading conditions, power balance within a single autonomous MG might not be met by the black start DGs with limited capacity. Even worse, customers that are initially restored might be dropped again to keep the system from losing synchronism. To keep the re-energized feeder from blackout again while having full use of MHK resources, the system operator could actively reconfigure the distribution network by forming dynamic MGs to pick up as much load as possible or mitigate power demand for DGs in lightly and/or heavily loaded areas. In particular, the coastal feeder could be actively reconfigured in response to the periodic generation of MHK resources; this produces an extended period during which the feeder is able to operate off-grid and maximizes customer restoration.

In this work, we propose a sequence of control actions to restore a coastal feeder experiencing a blackout in the context of dynamic MGs. The proposed restoration procedures are developed to restore a coastal feeder experiencing a blackout in the states where the whole feeder is ready to reconnect to the main grid. The main steps to be considered are identified into two stages:

- Stage I is designed to sectionalize the blackout feeder and independently re-energize as many sections as possible. During Stage I, the blackout system is predivided into min-MGs by the installed SSWs. Each min-MG is isolated from the external system using the opened SSWs and would black start itself independently using the locally installed black start units. No interactions among min-MGs are introduced at this stage and no MHK resources are reconnected. The whole system is managed separately by the smallest sets with minimum online assets.

- Stage II is designed to pick up as many customers and integrate as many MHK resources as possible, and to mitigate power demand by reconfiguring the network. Once sections of the system are operating autonomously, the isolated min-MGs would be sequentially interconnected and operated as a whole to enhance operational inertia during power oscillation from either load or MHK resources. The system topology would be varied in the context of the proposed dynamic MGs operational framework. Once the main grid is healthy, the islanded feeder could be operated synchronously with the main grid to achieve fast reconnection.

The following sequence of actions are defined to perform the proposed two-stage restoration procedure:

- The SSWs will automatically sectionalize the blackout feeder into isolated min-MGs. All the MHK resources and other non-dispatchable resources (e.g., photovoltaics [PVs]) should be disconnected at this step to avoid undesired power fluctuation. The distribution automation should also disconnect noncritical loads as much as possible to avoid overloading the black start unit.

- Once the feeder is properly sectionalized into min-MGs and minimum assets are connected, each min-MG would be re-energized to pick up the critical loads within. 
To ensure adjacent sections are isolated in case certain min-MGs fail to re-energize during this step, the SSWs should remain open. During the re-energization, all available black start units should be used in a coordinated manner.

- Once the min-MGs are properly re-energized and their operating frequencies and voltages are steady, the adjacent min-MGs would be actively interconnected to form dynamic MGs. The electric boundaries of these dynamic MGs could be actively varied to maximize the system's total load energization and MHK resource penetration.

- Once the main grid has recovered, the islanded feeder should be seamlessly reconnected to the main grid. At this step, the feeder operates as a single islanded $\mathrm{MG}$ and should be operated synchronously with the main grid to reconnect quickly and reliably.

Finally, we present the proposed two-stage restoration procedure for a blackout coastal feeder using dynamic MGs in Figure 3-3.

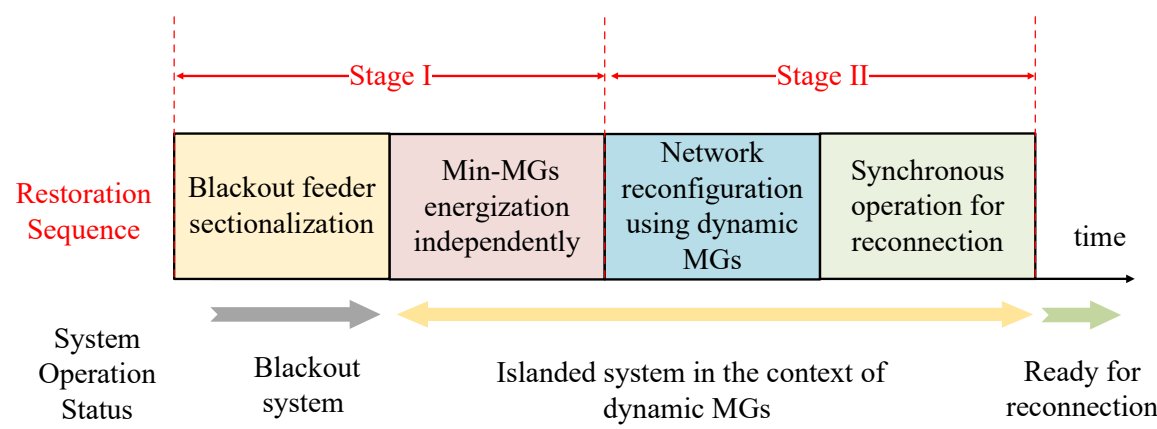

FIGURE 3-3 Two-stage Restoration Strategy for Coastal Feeder Using Dynamic MGs. 


\section{CASE STUDY}

Figure 4-1 shows how we establish a 13-bus test system of dynamic and networked MGs representing a coastal distribution feeder to validate the effectiveness of the proposed control strategy. The test system is comprised of three min-MGs, two SSWs, and six DGs. Among these six DGs, four (DGs 1, 3,5 and 6) are operated autonomously. MHK energy integration is considered, where DG 2 and DG 4 are used to represent wave and tidal generation, respectively. Note that when the time-scale of the sample wave generation profile from Figure 2-1 is in seconds, the power generated from tidal energy should be almost a constant value at the same time-scale. Therefore, in the two case studies below, the sample active power reference value of DG 4 is represented by one positive constant and one negative constant, respectively . Moreover, critical loads are identified at buses $3,5,8$, and $12\left(S_{\mathrm{L}}=S_{\mathrm{L} 3}=S_{\mathrm{L} 5}=S_{\mathrm{L} 8}=0.5 S_{\mathrm{L} 12}\right)$. Finally, the control gains are designed based on a general design criterion mentioned in the previous section. Detailed system parameters are presented in Table 4-1.

The dynamic MGs reconfiguration scheme is presented with the topology variation (topologies $1 \sim 6$ ), as shown in Figure 4-2. Under topology 1, the system initially operates in grid-connected mode with the main breaker and SSWs closed. Because a grid contingency (e.g., power outage) isolates the entire coastal distribution feeder from upstream power grids, the

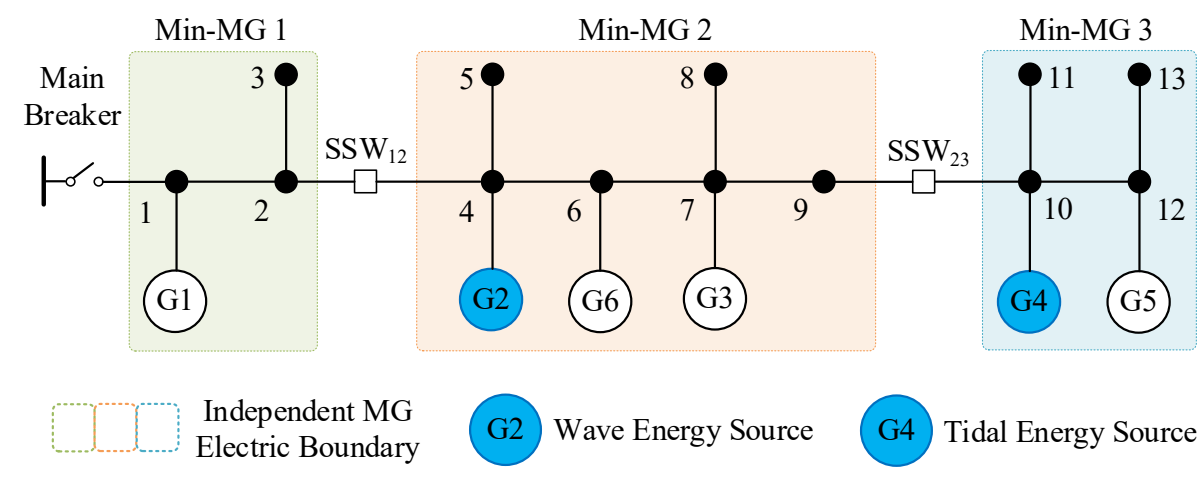

FIGURE 4-1 Topology of a 13-bus Test System.

TABLE 4-1 Parameters of Test System

\begin{tabular}{lccc}
\hline \multicolumn{1}{c}{ Parameter } & Symbol & Value & Unit \\
\hline DG Power & $P^{*}, Q^{*}$ & 100,100 & $\mathrm{~kW} / \mathrm{kVar}$ \\
Critical Load & $P_{\mathrm{L}}, Q_{\mathrm{L}}$ & 40,20 & $\mathrm{~kW} / \mathrm{kVar}$ \\
Internal Impedance & $R_{\mathrm{v}}, L_{\mathrm{V}}$ & $0,2.17$ & $\mathrm{~m} \Omega / \mathrm{mH}$ \\
Line Impedance & $R_{\mathrm{T}}, L_{\mathrm{T}}$ & $16.8,0.0535$ & $\mathrm{~m} \Omega / \mathrm{mH}$ \\
Control Gain & $k_{\mathrm{f}}, k_{\mathrm{p}}, k_{\mathrm{E}}, k_{\mathrm{Q}}$ & $8,1,5,80$ & - \\
& $\mu_{\mathrm{P}, \mathrm{XY}}, \mu_{\theta, \mathrm{XY}}$ & $5,3.7$ & - \\
& $\mu_{\mathrm{Q}, \mathrm{XY}}, \mu_{\mathrm{V}, \mathrm{XY}}$ & 200,20 & - \\
& $k_{\mathrm{U}}$ & 50 & - \\
& $\Delta t_{\mathrm{DG}}, \Delta t_{\mathrm{ssw}}$ & 100,100 & $\mathrm{~ms}$ \\
\hline
\end{tabular}


main breaker is open and dynamic MGs are activated for restoration; this leads the system to operate in islanded mode, and its topology changes from topology 1 to 2 . Moreover, to mitigate the impacts of grid contingency, the system is requested by the system operator to be actively and continuously reconfigured from topology 2 to 6 ; the system reconfiguration operations with SSWs reopening are shown under topologies 3 and 4 . The system reconfiguration options with SSWs reclosure are presented under topologies 5 and 6.

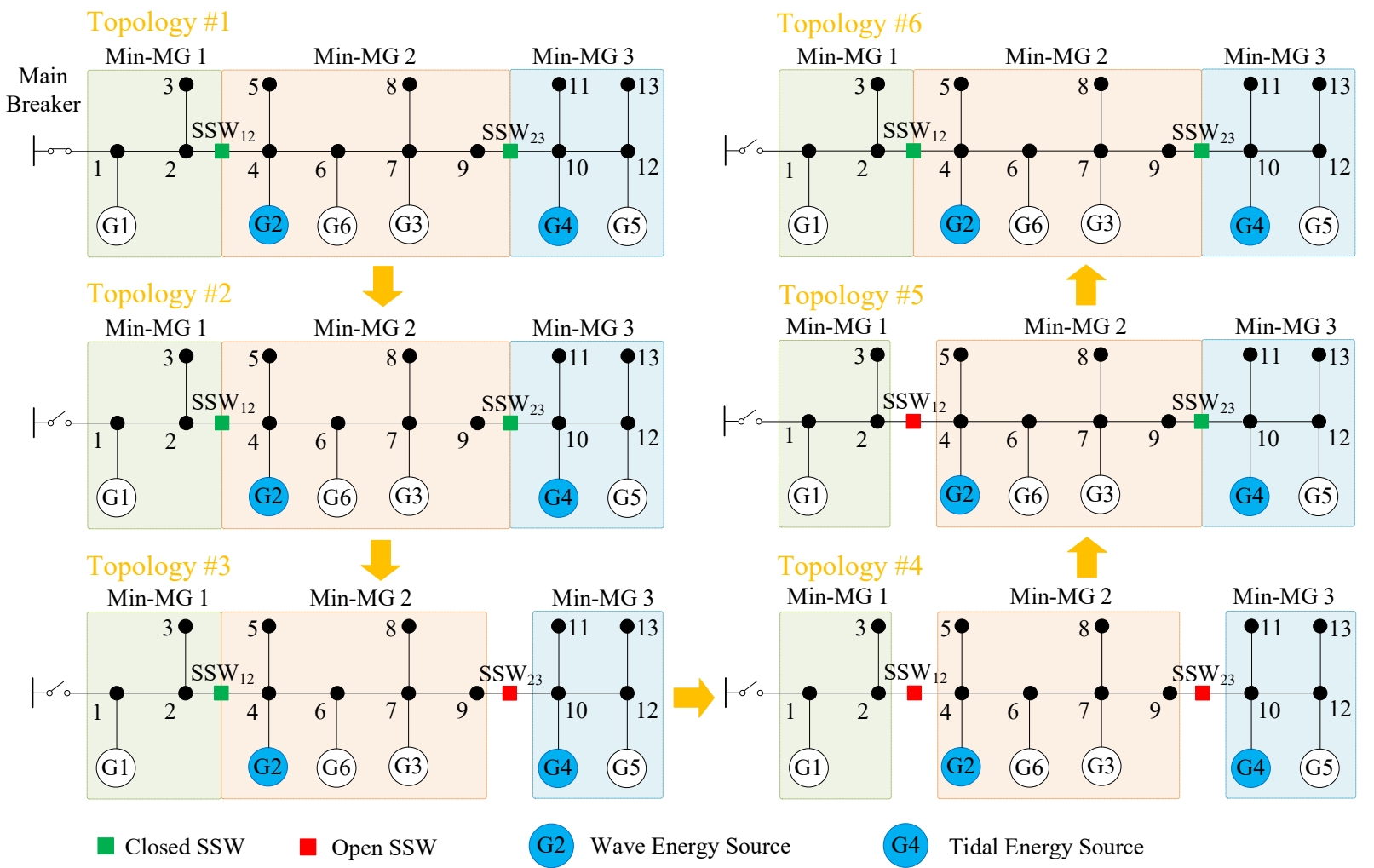

FIGURE 4-2 Dynamic MG Reconfiguration Schemes.

\subsection{CASE I: COASTAL DISTRIBUTION FEEDER RECONFIGURATION WITH POSITIVE TIDAL GENERATION}

As shown in Figure 4-2, the optimization-driven topology variation and the proposed control strategy are implemented in the context of dynamic and networked MGs. The active power reference of DG 2 is from the sample wave generation profile with respect to $t_{\text {final }}=60 \mathrm{~s}$, while the active power reference of DG 4 is given by the sample constant positive power $\left(P_{\text {ref4 }}=30 \mathrm{~kW}\right)$ generated by tidal energy.

The system initially operates under grid-connected mode with the main breaker and both SSWs closed, as depicted in topology 1 in Figure 4-2. The corresponding simulation test results are shown in Figure 4-3. Before the reconfiguration is requested $\left(t<t_{1}=4 \mathrm{~s}\right)$, the grid-connected 
system is regulated in Type I mode, where system frequency and voltage are maintained by the main grid and DG reactive power outputs are regulated using PI controller $\left(Q_{\text {ref }}=0\right)$ s.

Figure 4-2 presents the performance of the proposed controller without reconfiguration requests in topology 2, where the main breaker is open and both SSWs remain closed. As depicted in Figure 4-3, at $t=t_{1}=4 \mathrm{~s}$, the system operates in islanded mode with the proposed secondary controller under Type I mode activated. After the controller is activated $\left(t>t_{1}\right)$, system frequency and voltage are regulated as rated. Except for DG 2 and 4, whose power outputs follows the pre-defined wave and tidal power profiles, each MG achieves accurate proportional DG active and reactive power sharing.

Figure 4-3 details the proposed controller performance of system reconfiguration with SSW in topologies 3 and 4, where the system is requested by the system operator to reconfigure from topology 2 to topology 3 , and then to topology 4 . The requested reconfiguration is executed with the proposed controller under Type II activated, and system status is presented in Figure 43 .

As depicted in Figure 4-3, $\mathrm{SSW}_{23}$ receives the reconfiguration request at $t=t_{2}=8 \mathrm{~s}$ and changes its status at $t=t_{3}=12 \mathrm{~s}$, while the $\mathrm{SSW}_{12}$ remains closed. With the proposed control, the power flowing through $\mathrm{SSW}_{23}$ is minimized before the reconfiguration. No significant transients are introduced at $t=t_{3}$, and a seamless system transition is achieved. Furthermore, $\mathrm{SSW}_{12}$ is requested to open at $t=t_{4}=14 \mathrm{~s}$, while $\mathrm{SSW}_{23}$ remains open. Then the proposed controller switches to Type II mode, the system transits seamlessly as $\mathrm{SSW}_{12}$ opens at $t=t_{5}=17.4 \mathrm{~s}$, and the requested reconfiguration is achieved. No significant transients are introduced during the reconfiguration.

Figure 4-2 illustrates the performance of the proposed controller during system reconfiguration with SSW re-closure in topologies 5 and 6 , where the system is requested to reconfigure from topology 4 to topology 5 , and then to topology 6 . The requested reconfiguration is executed with the proposed controller, with Type III activated. System status is presented in Figure 4-3.

As shown in Figure 4-3, $\mathrm{SSW}_{23}$ receives a request at $t=t_{6}=18 \mathrm{~s}$ and changes status at $t=t_{7}=23.4 \mathrm{~s}$. With the proposed controller, Type III mode is activated at $t=t_{6}$ and the voltage phasors are synchronized before reconfiguration. No significant transients are introduced at $t=t_{6}$ and a seamless system transition is achieved. After the reconfiguration request is fulfilled $\left(t>t_{7}\right)$, the proposed controller switches back to Type I mode and regroups the DGs accordingly. Figure 4-3 shows that proportional power sharing among DGs 3, 5, and 6 in the new group is achieved. However, when $t=t_{8}=30 \mathrm{~s}, \mathrm{SSW}_{12}$ is requested to re-close and change its status at $t=t_{9}=36.5 \mathrm{~s}$. Then the proposed controller switches to Type III mode. The system transits seamlessly as $\mathrm{SSW}_{12}$ re-closes at $t=t_{9}$, and the requested reconfiguration is achieved. No significant transients are introduced during reconfiguration. After the reconfiguration request is satisfied $\left(t>t_{9}\right)$, the proposed controller switches back to Type I mode. Figure 4-3 shows that proportional power sharing among DGs 1, 3, 5, and 6 is achieved. 

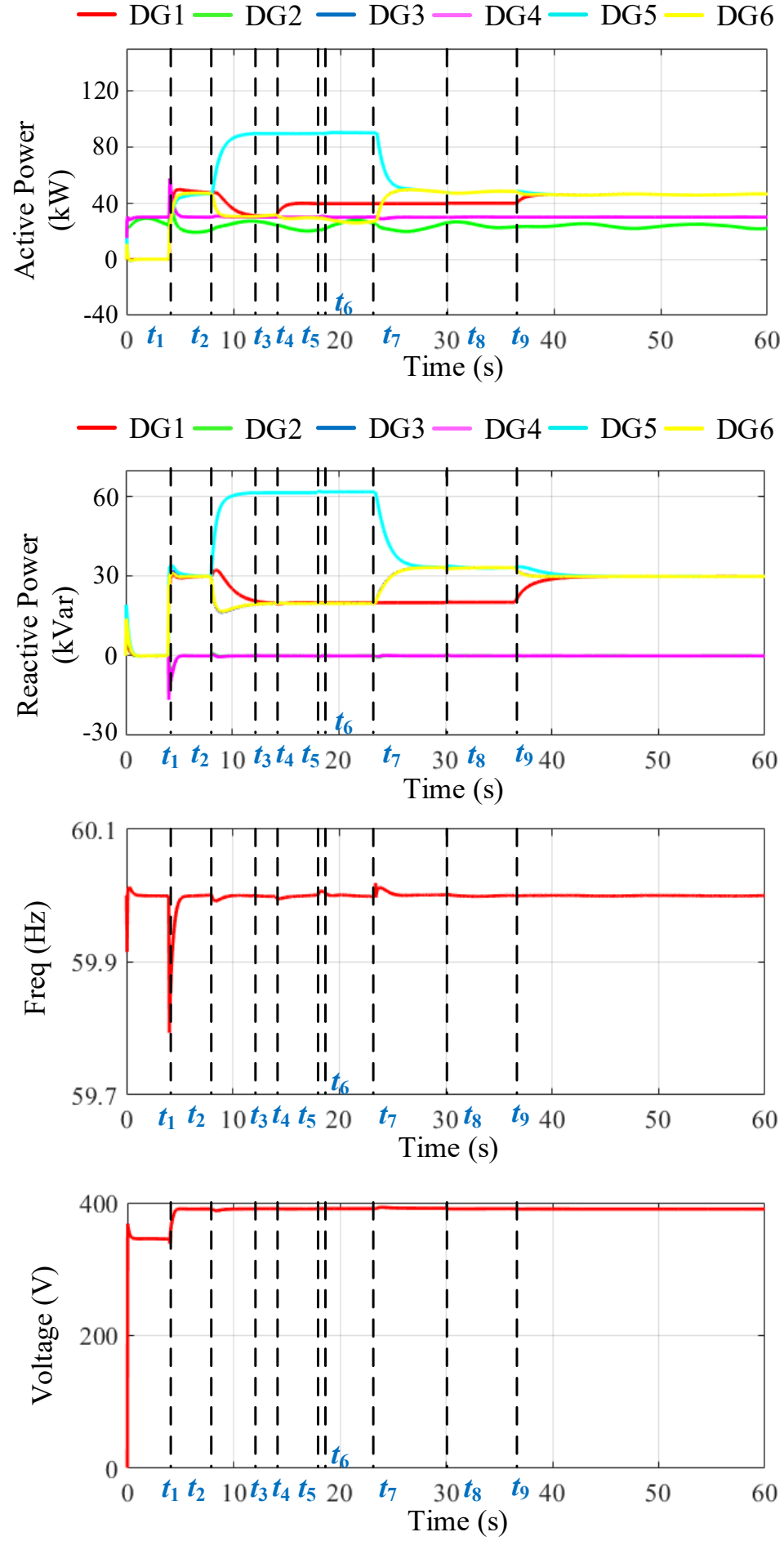

FIGURE 4-3 Simulation Results for System Reconfiguration in Case I. 


\subsection{CASE II: COASTAL DISTRIBUTION FEEDER RECONFIGURATION WITH NEGATIVE TIDAL GENERATION}

In this case, similar to Case I, the dynamic MG reconfiguration is presented with topology variations in Figure 4-2, driven by the optimization algorithm. Distributed controls detailed in the previous sections are used to ensure steady and transient state operation. The sample wave generation profile is utilized as the active power reference of DG 2 in terms of $t_{\text {final }}$ $=60 \mathrm{~s}$, while the active power reference of DG 4 is given by the constant negative power $\left(P_{\text {ref } 4}=\right.$ $-30 \mathrm{~kW}$ ) generated by tidal energy. The real-time system status with the proposed controller is shown in Figure 4-4.

As depicted in topology 1 in Figure 4-2, at the initial stage the system operates in grid-connected mode and all the main breaker and SSWs are closed. When $t<t_{1}=4 \mathrm{~s}$, the grid-connected system is regulated in Type I mode. Further, in Figure 4-2, topology 2 shows the proposed controller performance for system operation without reconfiguration. When $t=t_{1}=4 \mathrm{~s}$ in Figure 4-4, the system operates in islanded mode with Type I mode activated. After $t>t_{1}$, system frequency and voltage are regulated as rated. Except for DGs 2 and 4 with power profiles representing MHK energy, accurate proportional DG active and reactive power sharing is achieved within each MG.

Topologies 3 and 4 in Figure 4-2 illustrate the proposed controller performance for system reconfiguration with SSW opening. The requested reconfiguration is executed with the proposed controller under Type II activated. As shown in Figure 4-4, $\mathrm{SSW}_{23}$ first receives the reconfiguration request at $t=t_{2}=8 \mathrm{~s}$, where the power flowing through $\mathrm{SSW}_{23}$ is eliminated before reconfiguration. No significant transients are shown at $t=t_{3}=12 \mathrm{~s}$. Next, $\mathrm{SSW}_{12}$ is requested to open at $t=t_{4}=14 \mathrm{~s}$, and the power flowing through $\mathrm{SSW}_{12}$ is eliminated before reconfiguration. No significant transients are observed at $t=t_{5}=17.4 \mathrm{~s}$.

Topologies 5 and 6 in Figure 4-2 show the proposed controller performance for system reconfiguration with SSW closure. The requested reconfiguration is executed with Type III activated. As shown in Figure 4-4, $\mathrm{SSW}_{23}$ first receives a request at $t=t_{6}=18 \mathrm{~s}$ and changes status at $t=t_{7}=23.4 \mathrm{~s}$. No significant transients are observed at $t=t_{6}$, and a seamless system transition is achieved. After $t>t_{7}$, the proposed controller switches back to Type I mode and proportional power sharing among DGs 3,5 , and 6 is achieved. Next, $t=t_{8}=30 \mathrm{~s}, \mathrm{SSW}_{12}$ is requested to re-close and change its status at $t=t_{9}=36.5 \mathrm{~s}$. Then the system transits seamlessly with Type III mode activated as $\mathrm{SSW}_{12}$ re-closes at $t=t_{9}$. After $t>t_{9}$, the proposed controller switches back to Type I mode and power is shared proportionally among DGs 1, 3, 5, and 6 . 

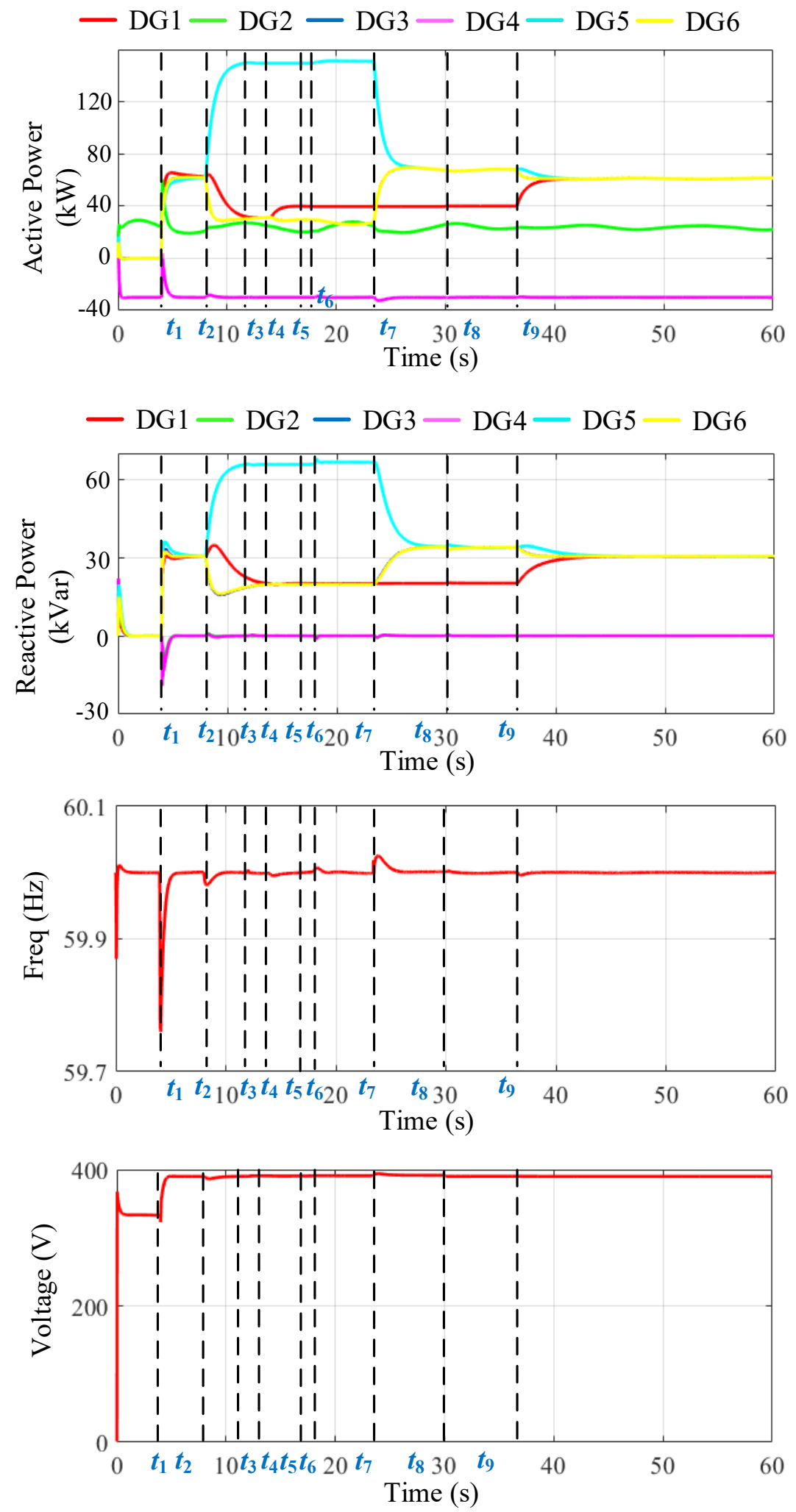

FIGURE 4-4 Simulation Results for System Reconfiguration in Case II. 


\section{SUMMARY AND CONCLUSION}

The resilient operation of the coastal community is an important topic for grid modernization. Extreme events caused by climate change have recently challenged the operational resiliency of coastal feeders; however, the coastal community is motivated in integrating renewable resources (e.g., MHK resources) that would help mitigate extreme event risks. MHK resources have different operational characteristics than the renewable resources that are installed inland, which results in additional challenges for coastal feeder operation.

During extreme events when the substations are unavailable, the coastal feeders could operate autonomously by forming network MGs. Dynamic MG is an advanced structure of network MG. Compared to the conventional definition of MG, where the network topology is predetermined and fixed, the electric boundaries of dynamic MGs could vary per the system operator's request. The coastal feeders could be actively reconfigured in the context of dynamic MG operation with enhanced resiliency. However, unlike a conventional power system with large inertia, coastal feeders are energized by inverter-interfaced DGs and are more vulnerable to undesired system transients during network reconfiguration. Successful network reconfiguration of dynamic MGs should be done seamlessly, which requires additional effort.

In this work, we propose a holistic optimization and control framework for coastal feeder operation in the context of dynamic and networked MGs. The concept of a min-MG is introduced as the building block of dynamic MGs. The whole feeder is sectionalized into multiple adjacent min-MGs by industry-approved SSWs. The boundaries of the min-MGs are determined by the SSWs within; the interconnected min-MGs would operate as a whole and form dynamic MGs. The topology variation of dynamic MGs is fulfilled by turning the corresponding SSWs on and off as requested. To fully exploit the operational flexibility created by operating MGs dynamically, the cybernetwork of the proposed control framework is also reconfigurable and the feeder would operate as a coupled CPS.

A set of secondary controllers is designed for inverter-interfaced DGs with the capability to form grids so that MGs can operate dynamically and resiliently. In addition to constant frequency and/or voltage regulations, the controller we propose would enable seamless transitions of system topology and proportional power-sharing among DGs that are physically connected. The proposed controller uses distributed control, which further enhances the system's operational resiliency by avoiding single point of failure. No centralized controller and dedicated communication network are needed.

We also derived sufficient conditions under which the system with the proposed controller is exponentially stable. Furthermore, we analyzed the use of the proposed dynamic MGs control framework to restore blackout coastal feeders. Compared to conventional black start techniques, the participation of dynamic MGs in grid service restoration provides the system operator with more feasible options. 


\section{ACKNOWLEDGEMENT}

The technical report is based upon work supported by the U.S. Department of Energy's Office of Energy Efficiency and Renewable Energy (EERE) under the Water Power Technologies Office Seedling Project Award Number 36321 and the Solar Energy Technologies Office Award Number 34230. 


\section{REFERENCES}

[1] R. He et al., "Gulf stream marine hydrokinetic energy resource characterization off Cape Hatteras, North Carolina USA," in OCEANS 2016 - Shanghai, 2016, pp. 1-4.

[2] J. Ward, I. Schultz, D. Woodruff, G. Roesijadi, and A. Copping, "Assessing the effects of marine and hydrokinetic energy development on marine and estuarine resources," in OCEANS 2010 MTS/IEEE SEATTLE, 2010, pp. 1-7.

[3] R. Manasseh, S. A. Sannasiraj, K. L. McInnes, V. Sundar, and P. Jalihal, "Integration of wave energy and other marine renewable energy sources with the needs of coastal societies," Int. J. Ocean Clim. Syst., vol. 8, no. 1, pp. 19-36, 2017.

[4] "IEEE Application Guide for IEEE Std 1547, IEEE Standard for Interconnecting Distributed Resources with Electric Power Systems," IEEE Std 1547.2-2008, pp. 1-217, 2009.

[5] C. Chen, J. Wang, and D. Ton, "Modernizing Distribution System Restoration to Achieve Grid Resiliency Against Extreme Weather Events: An Integrated Solution," Proc. IEEE, vol. 105, no. 7, pp. 1267-1288, 2017, doi: 10.1109/JPROC.2017.2684780.

[6] D. T. Ton and M. A. Smith, "The U.S. Department of Energy's microgrid initiative," Electr. J., vol. 25, no. 8, pp. 84-94, 2012.

[7] M. E. Nassar and M. M. A. Salama, "Adaptive self-adequate microgrids using dynamic boundaries," IEEE Trans. Smart Grid, vol. 7, no. 1, pp. 105-113, 2016.

[8] Y. Du, X. Lu, J. Wang, and S. Lukic, "Distributed Secondary Control Strategy for Microgrid Operation with Dynamic Boundaries," IEEE Trans. Smart Grid, vol. 10, no. 5, p. 1, 2018, doi: 10.1109/TSG.2018.2879793.

[9] J. Li, X. Ma, C. Liu, and K. P. Schneider, "Distribution System Restoration with Microgrids Using Spanning Tree Search," IEEE Trans. Power Syst., vol. 29, no. 6, pp. 3021-3029, 2014.

[10] Y. Xu, C. C. Liu, K. P. Schneider, F. K. Tuffner, and D. T. Ton, "Microgrids for service restoration to critical load in a resilient distribution system," IEEE Trans. Smart Grid, vol. 9, no. 1, pp. 426-437, 2018, doi: 10.1109/TSG.2016.2591531.

[11] F. Tang, J. M. Guerrero, J. C. Vasquez, D. Wu, and L. Meng, "Distributed active synchronization strategy for microgrid seamless reconnection to the grid under unbalance and harmonic distortion," IEEE Trans. Smart Grid, vol. 6, no. 6, pp. 2757-2769.

[12] Y. Kim, J. Wang, and X. Lu, “A Framework for Load Service Restoration Using Dynamic Change in Boundaries of Advanced Microgrids with Synchronous-Machine DGs," IEEE Trans. Smart Grid, vol. 9, no. 4, pp. 3676-3690, 2018. 
[13] Y. Ma et al., "Real-Time Control and Operation for a Flexible Microgrid with Dynamic Boundary," in 2018 IEEE Energy Conversion Congress and Exposition (ECCE), 2018, pp. 5158-5163.

[14] A. Mohsenzadeh, C. Pang, and M. Haghifam, "Determining Optimal Forming of Flexible Microgrids in the Presence of Demand Response in Smart Distribution Systems," IEEE Syst. J., vol. 12, no. 4, pp. 3315-3323, 2018.

[15] Y. Du, X. Lu, H. Tu, J. Wang, and S. Lukic, "Dynamic Microgrids With Self-Organized Grid-Forming Inverters in Unbalanced Distribution Feeders," IEEE J. Emerg. Sel. Top. Power Electron., vol. 8, no. 2, pp. 1097-1107, 2020.

[16] Y. Du, X. Lu, J. Wang, B. Chen, H. Tu, and S. Lukic, "Dynamic Microgrids in Resilient Distribution Systems with Reconfigurable Cyber-Physical Networks," IEEE J. Emerg. Sel. Top. Power Electron., p. 1, 2020.

[17] A. Blavette, D. L. O’Sullivan, R. Alcorn, T. W. Lewis, and M. G. Egan, "Impact of a Medium-Size Wave Farm on Grids of Different Strength Levels," IEEE Trans. Power Syst., vol. 29, no. 2, pp. 917-923, 2014.

[18] A. Blavette, "Grid integration of wave energy and generic modelling of ocean devices for power system studies," 2013.

[19] G. Hagerman and B. Polagye, "Methodology for estimating tidal current energy resources and power production by tidal in-stream energy conversion (TISEC) devices," Electr. Power Res. Inst., 2006.

[20] R. Thresher, "A commercialization path and challenges for marine hydrokinetic renewable energy," in 2011 IEEE Power and Energy Society General Meeting, 2011, pp. $1-8$.

[21] R. Manasseh, S. Sannasiraj, K. L. McInnes, V. Sundar, and P. Jalihal, "Integration of wave energy and other marine renewable energy sources with the needs of coastal societies," Int. J. Ocean Clim. Syst., vol. 8, no. 1, pp. 19-36, 2017.

[22] J. M. Guerrero, J. C. Vasquez, J. Matas, L. G. De Vicuña, and M. Castilla, "Hierarchical control of droop-controlled AC and DC microgrids: A general approach toward standardization," IEEE Trans. Ind. Electron., vol. 58, no. 1, pp. 158-172, 2011.

[23] Y. Du, "Distributed Control of Networked Microgrids with Dynamic Boundaries (Doctoral dissertation, North Caronlina State University, Raleigh, US)," 2019.

[24] S\&C Electric Company, "IntelliRupter® PulseCloser Fault Interrupter."

[25] J. Guo, W. Liu, F. R. Syed, and J. Zhang, "Reliability assessment of a cyber physical microgrid system in island mode," CSEE J. Power Energy Syst., vol. 5, no. 1, pp. 46-55, 2019. 
[26] Z. Cheng, J. Duan, and M. Chow, "To Centralize or to Distribute: That Is the Question: A Comparison of Advanced Microgrid Management Systems," IEEE Ind. Electron. Mag., vol. 12, no. 1, pp. 6-24, 2018.

[27] J. W. Simpson-Porco, Q. Shafiee, F. Dörfler, J. C. Vasquez, J. M. Guerrero, and F. Bullo, "Secondary frequency and voltage control of islanded microgrids via distributed averaging," IEEE Trans. Ind. Electron., vol. 62, no. 11, pp. 7025-7038.

[28] Y. Du, X. Lu, B. Chen, J. Liu, X. Wang, and F. Blaabjerg, "Distributed Average Observation in Inverter Dominated Dynamic Microgrids," in 2020 IEEE Energy Conversion Congress and Exposition (ECCE), 2020, pp. 4627-4633.

[29] R. A. Horn, R. A. Horn, and C. R. Johnson, Matrix analysis. Cambridge University Press, 1990.

[30] J. Feltes and C. Grande-Moran, "Down, but Not Out: A Brief Overview of Restoration Issues," IEEE Power Energy Mag., vol. 12, no. 1, pp. 34-43, 2014.

[31] "IEEE Standard for the Specification of Microgrid Controllers," IEEE Std 2030.7-2017, pp. 1-43, 2018.

[32] L. Che, M. Khodayar, and M. Shahidehpour, "Only Connect: Microgrids for Distribution System Restoration,” IEEE Power Energy Mag., vol. 12, no. 1, pp. 70-81, 2014. 



\section{Argonne}

\section{Energy Systems Division}

Argonne National Laboratory

9700 South Cass Avenue, Bldg. 362

Lemont, IL 60439-4854

www.anl.gov 